\title{
Massless Composite Bosons Formed by the Coupled Electron-Positron Pairs and Two-Photon Angular Correlations in the Colliding Beam Reaction $e^{-} e^{+} \rightarrow B \gamma \gamma$ with Emission of the Massless Boson
}

\author{
A. I. Agafonov 1 \\ National Research Centre "Kurchatov Institute", Moscow 123182, Russia \\ Correspondence should be addressed to A. I. Agafonov; Agafonov_AIV@nrcki.ru
}

Received 30 October 2017; Revised 25 February 2018; Accepted 26 April 2018; Published 24 June 2018

Academic Editor: Juan José Sanz-Cillero

Copyright (C) 2018 A. I. Agafonov. This is an open access article distributed under the Creative Commons Attribution License, which permits unrestricted use, distribution, and reproduction in any medium, provided the original work is properly cited. The publication of this article was funded by SCOAP $^{3}$.

\begin{abstract}
The approach in which the electron and positron are treated as ordinary, different particles, each being characterized by the complete set of the Dirac plane waves, is examined. This completely symmetric representation that is beyond the standard QED makes it necessary to choose another solution of the Dirac equation for the free particle propagator as compared to that used currently. The Bethe-Salpeter equation with these particle propagators is solved in the ladder approximation. A new solution has been found represented by the massless composite bosons formed by the coupled electron-positron pairs with the coupling equal to the fine structure constant. It has been demonstrated that (1) the massless boson states have normalizable complex wave functions which are transversely compressed plane waves; (2) the transverse radius of the wave functions diverges as the boson energy goes to zero; that is, the composite bosons cannot be at rest; (3) increasing the boson energy results in an extension of the transverse wave function in the momentum space and a corresponding contraction of the real space coordinate wave function. The new reaction $e^{-} e^{+} \rightarrow B \gamma \gamma$ is investigated with the products composed of the massless composite boson and two photons. The cross-section of this reaction is derived for nonrelativistic colliding beams of spin-polarized electrons and positrons. In this case the $2 \gamma$ angular correlation spectrum is characterized by a narrow peak with the full-width-at-half-maximum not exceeding $0.2 \mathrm{mrad}$. It is shown that in order to distinguish between the conventional annihilation of the singlet electron-positron pair with the two-photon emission and the new examined reaction yielding the three particles, experiments are proposed with the extremely nonrelativistic colliding beams.
\end{abstract}

\section{Introduction}

During the development of the QED theory, Feynman considered essentially two possible ways $[1,2]$. In the first way, assuming that the fermion-antifermion symmetry must exist in nature, Feynman derived the free fermion propagator (Equation (17) in [1]). In this propagator the negative-energy states are assumed to be not available to the electrons; the upper continuum is assumed to be not available to the positrons which are recognized as particles traveling backwards in time [2]. The total number of degrees of freedom that is determined by the complete basis of the Dirac plane waves [3] is divided into half. One half of the degrees of freedom is assigned to the electron and the other to the positron. The modern description of the electron-positron field is based on this propagator.

Note that, in many situations, the filled electronic states with negative energies cannot be ignored. So, these states play a prominent role in the behavior of an electron in external fields, for example, in the Coulomb field $[4,5]$. The filled lower continuum is important in the analysis of the electronic structure in super-heavy nuclei, for which at a certain nuclear charge the electron lower level $1 S_{1 / 2}$ merges with the bottom of the lower continuum $[6,7]$. A similar situation arises in discussion of the value of the cosmological constant. Apart from the positive contribution from the zero-point energy of 
boson quantized fields, another energy source is derived from the Dirac theory of the electron because the filled levels lead inevitably to negative contribution to the vacuum energy [8]. In a study of the radiation scattering by free electrons it was concluded that radiation-induced electron quantum jumps in the intermediate states of negative energies are crucial for the scattering [9].

Taking into account the contradictory situation presented above, in the present paper we have tried to find out what one can expect from the second way that was also discussed by Feynman [1], but has not been studied so far. In this second way Feynman also proposed the free electron propagator, in which the electron is characterized by the complete set of the Dirac plane waves [3]. In this case there is the only possibility of treating the electron and the positron as independent particles and of using a similar propagator for the positron. Then both the electron and the positron are characterized by the number of degrees of freedom that exactly corresponds to the Dirac theory. There is no reason to doubt that the complete spectrum of states for any system of interacting particles can be deduced only when the full basis of states is taken into account for each particle of the system. According to our considerations the division of the complete planewave basis into two parts as discussed above leads to the following fact: neither electron states nor positron states separately form the complete system of the wave functions. Therefore, the full coupled electron-positron system may possess additional states as compared to the partial electron and positron subsystems.

The electron-positron field theory derived from the hole theory of positrons [1] leads to a clear picture of the annihilation process of electron-positron pairs. In this process nothing remains of the electron and positron, and the reaction products are just a few photons $[1,3,5,10]$. The singlet pair of free particles with the center of mass at rest is converted with the greatest probability into two photons which, due to the momentum conservation, should be emitted exactly in the two opposite directions, at the angle of $180^{\circ}$ to each other.

Below we consider only reactions of low-energy electronpositron pairs. This article does not take into account any high-energy electron-positron reactions, when the reaction products can be either charged leptons (electrons, muons, taus) or hadrons [11-18]. In the case of the existence of the predicted massless composite bosons there should be a process that is, in a sense, similar to the conventional process of electron and positron annihilation, but it has a fundamental difference from the latter. The reaction products in this new process involve, together with emitted photons, the massless boson which is formed by the strongly coupled electron-positron pair. This annihilation-like process with emission of two photons can be represented as follows:

$$
e^{-} e^{+} \longrightarrow B \gamma_{1} \gamma_{2}
$$

where $B$ denotes the massless composite boson. It is crucial for this reaction that the two-photon angular correlation spectra must have finite angular widths even for the electronpositron pairs with the center of mass at rest. This is due to the fact that there are three particles in the reaction products. To prove the existence of the third body in the reaction outcome it is of fundamental importance to find the angular width of the $2 \gamma$ correlation spectra.

The article consists of two parts. The first part, presented in Section 2, is devoted to derivation of the massless boson states formed by the coupled electron-positron system with the actual coupling equal to the fine structure constant. Results obtained for the massless boson wave functions are presented. The goal of the second part presented in Section 3 of the paper is to study the cross-section of the reaction (1) for the extremely nonrelativistic colliding beams of the electrons and positrons. The minimal angular width of the $2 \gamma$ correlation spectrum is obtained numerically. In addition, in Section 4 experiments are suggested to establish whether there is the conventional annihilation of singlet electronpositron pair with the two-photon emission or the proposed new reaction with the three-particle outcome. Note that such experiments constitute a relatively simple possibility of testing the central particle-antiparticle concept of the Standard Model.

Natural units $(\hbar=c=1)$ will be used throughout.

\section{The Massless Composite Bosons}

2.1. The Free Fermion Propagator. At present in QED the free propagator for the Dirac equation is used in the following form $[1,4,5]$ :

$$
\begin{aligned}
K_{+}(2,1)= & \sum_{\mathbf{p}} \psi_{p}(2) \bar{\psi}_{p}(1) \theta\left(t_{2}-t_{1}\right) \\
& -\sum_{\mathbf{p}} \psi_{-p}(2) \bar{\psi}_{-p}(1) \theta\left(t_{1}-t_{2}\right) .
\end{aligned}
$$

Here $\psi_{ \pm p}$ is the Dirac plane wave representing the state of the free particle with energy $\pm \varepsilon_{p}$, respectively, and $\bar{\psi}_{p}$ the Dirac conjugate wave function. In (2) the contribution to $K_{+}(2,1)$ at $t_{2}>t_{1}$ is due to the electron terms and at $t_{2}<t_{1}$ to the positron terms.

The Bethe-Salpeter equation $[19,20]$ with the propagator (2) was studied in many works, as a rule, in the ladder approximation [21]. After the work [22], considerable attention is given to the problem of strongly coupled states for fermionantifermion systems. In the most commonly used approach to the problem the Bethe-Salpeter equation is regarded as eigenvalues task for the coupling constant [23-30]. That is, an eigenvalue is considered as the necessary strength of the attractive potential to make a massless bound state.

The Dirac equation, as well as any differential equation, has several solutions for the Green function [31]. The Green function for the Dirac equation can also be presented in the following form:

$$
\begin{aligned}
K_{-} & (2,1) \\
& =\sum_{\mathbf{p}}\left(\psi_{p}(2) \bar{\psi}_{p}(1)+\psi_{-p}(2) \bar{\psi}_{-p}(1)\right) \theta\left(t_{2}-t_{1}\right) .
\end{aligned}
$$

Both the propagators (2) and (3) were discussed in [1]. In the positron hole theory that is the foundation of QED, the 
negative-energy states are assumed to be not available to the electron. Therefore from this point of view the choice of (3), for which the negative-energy states are available for the electron, is unsatisfactory, as noted in [1].

On the other hand, the situation changes radically when the consideration is based on the Dirac theory. Because the electron must be characterized by the total number of degrees of freedom, the propagator (2) is not suitable, and the propagator (3), in which all of the spectrum of the Dirac plane waves is taken into account, should be applied. There is no doubt that the positron can be described by the Dirac equation as well. Considering (3) as the electron propagator, the only opportunity to use a free propagator similar to (3) for the positron is to assume that the electron and positron are independent particles.

In this approach, in the vacuum state, the lower continua for each of these particles are completely filled and the upper continua are not occupied. Then this vacuum state is charge-neutral and stable. The latter is due to the fact that the annihilation of the electrons and the positrons in the negative-energy states is forbidden by the energy conservation law since $\delta\left(\varepsilon_{\mathrm{el}}+\varepsilon_{\text {pos }}-\sum\right.$ the photon energies $) \equiv$ 0 when both the electron energy $\varepsilon_{\text {el }}$ and the positron energy $\varepsilon_{\text {pos }}$ are negative.

The interaction between electron and positron is attractive. In the ladder approximation the retarded interaction function can be written as follows [32]:

$$
\begin{aligned}
& G^{(1)}(3,4 ; 5,6) \\
& \quad=-e^{2}\left(1-\boldsymbol{\alpha}_{-} \boldsymbol{\alpha}_{+}\right) \delta_{+}\left(s_{56}^{2}\right) \delta(3,5) \delta(4,6) .
\end{aligned}
$$

Here $\boldsymbol{\alpha}_{ \pm}=\left(\begin{array}{cc}0 & \sigma_{ \pm} \\ \sigma_{ \pm} & 0\end{array}\right)$ are the velocity operators of the electron $(-)$ and positron $(+), \sigma_{ \pm}$are the Pauli matrices, and $s_{56}$ is the invariant distance between the particles.

This kind of the interaction function (4) is convenient for the subsequent development in this paper but at the same time we must exclude the $\beta$ matrices from the definition of the free fermion propagator (3). By doing this, in the approach of the absolutely symmetric representation of the particles, the electron propagator should be written as

$$
\begin{aligned}
& K_{0-}(2,1) \\
& =\sum_{\mathbf{p}}\left(\psi_{p}(2) \psi_{p}^{+}(1)+\psi_{-p}(2) \psi_{-p}^{+}(1)\right) \theta\left(t_{2}-t_{1}\right),
\end{aligned}
$$

and, similarly, for the positron propagator

$$
\begin{aligned}
& K_{0+}(4,3) \\
& =\sum_{\mathbf{p}}\left(\varphi_{p}(4) \varphi_{p}^{+}(3)+\varphi_{-p}(4) \varphi_{-p}^{+}(3)\right) \theta\left(t_{4}-t_{3}\right) .
\end{aligned}
$$

Here $\psi_{ \pm p}$ and $\varphi_{ \pm p}$ are the Dirac plane waves for the free electrons and positrons and $\psi_{ \pm p}^{+}$and $\varphi_{ \pm p}^{+}$are the Hermitian conjugate matrices with respect to $\psi_{ \pm p}$ and $\varphi_{ \pm p}$. The latter are given by $\psi_{p}, \varphi_{p}=u_{p, \pm} e^{-i p x}$ and $\psi_{-p}, \varphi_{-p}=u_{-p, \pm} e^{i p x}$, where

$$
\begin{aligned}
& u_{p, \pm}=\frac{1}{\sqrt{2 \varepsilon_{p}}}\left(\begin{array}{c}
\sqrt{\varepsilon_{p}+m} w_{ \pm} \\
\sqrt{\varepsilon_{p}-m}\left(\mathbf{n} \sigma_{ \pm}\right) w_{ \pm}
\end{array}\right), \\
& u_{-p, \pm}=\frac{1}{\sqrt{2 \varepsilon_{p}}}\left(\begin{array}{c}
\sqrt{\varepsilon_{p}-m}\left(\mathbf{n} \sigma_{ \pm}\right) w_{ \pm}^{\prime} \\
\sqrt{\varepsilon_{p}+m} w_{ \pm}^{\prime}
\end{array}\right),
\end{aligned}
$$

and $\boldsymbol{n}$ is the unit vector $\boldsymbol{n}=\boldsymbol{p} / \boldsymbol{p}$.

Considering (4)-(7), in the ladder approximation the bound-state Bethe-Salpeter equation for the electronpositron system is

$$
\begin{gathered}
\psi(1,2)=-i \iiint \int d \tau_{3} d \tau_{4} d \tau_{5} d \tau_{6} K_{0-}(1,3) K_{0+}(2,4) \\
\cdot G^{(1)}(3,4 ; 5,6) \psi(5,6),
\end{gathered}
$$

where $d \tau_{i}=d \boldsymbol{r}_{i} d t_{i}$

Below we do not consider the positronium states. Note only that in the nonrelativistic limit in which one neglects the interaction retardation and the interaction through the vector potential and assumes that the characteristic velocity of particles in the bound pair is much smaller than the speed of light, (8) with the propagators (5)-(6) is reduced to the Schrodinger equation for the Ps states.

2.2. The Boson Wave Function. We search for a solution $\psi(1,2)$ of $(8)$ in the form of a stationary wave with the phase velocity equal to the speed of light. Let $\boldsymbol{p}+\boldsymbol{q}=\boldsymbol{g}(\boldsymbol{p}$ and $\boldsymbol{q}$ are the momentum of the electron and positron, respectively), and the momentum of the pair, $\boldsymbol{g}$, is directed along the $z$ axis. It is the strongly coupled state with the momentum dependence of the boson energy $E=g$ that is valid only for massless particles. Due to the symmetry of the problem, we have to put $z_{1}=z_{2}=z$ and $t_{1}=t_{2}=t$ for this massless boson state that allows us to introduce a two-dimensional relative vector between the particles, $\boldsymbol{\rho}=\boldsymbol{\rho}_{1}-\boldsymbol{\rho}_{2}$. Then the wave function is

$$
\psi(1,2)=\varphi(\boldsymbol{\rho}, \mathbf{g}) \exp (i g(z-t)) .
$$

One can imagine (9) as transversely compressed plane wave. The wave cross-section is determined by the wave function of the transverse motion of the coupled pair $\varphi(\boldsymbol{\rho}, \boldsymbol{g})$, which should be normalizable:

$$
\int|\varphi(\boldsymbol{\rho}, \mathbf{g})|^{2} d \boldsymbol{\rho}=1
$$

For the state (9) the particle distribution is stationary and depends only on $\boldsymbol{\rho}$.

The function $\delta_{+}\left(s_{56}^{2}\right)$ in (4) was given in [32]. In our case it can be written as

$$
\begin{aligned}
& \delta_{+}\left(t_{56}^{2}-\rho_{56}^{2}\right) \\
& =\frac{1}{4 \pi \rho_{56}} \int_{-\infty}^{+\infty}\left(e^{-i \omega\left(t_{5}-t_{6}\right)}+e^{-i \omega\left(t_{6}-t_{5}\right)}\right) e^{i|\omega| \rho_{56}} d \omega,
\end{aligned}
$$

where $\rho_{56}=\left|\boldsymbol{\rho}_{5}-\boldsymbol{\rho}_{6}\right|$. It was taken into account that since the phase velocity of the wave (9) is equal to the velocity of light, 
the interaction between the electron and positron can only occur in the same layers $\left(z_{5}=z_{6}\right)$, which are perpendicular to the wave vector $\boldsymbol{g}$. Considering that $\alpha_{-z} \alpha_{+z}=1$ (here $\alpha_{-z}$ and $\alpha_{+z}$ are the $z$-components of the velocity operators for the electron and positron, respectively), the factor of (1 $\left.\boldsymbol{\alpha}_{-} \boldsymbol{\alpha}_{+}\right)$in (4) should be replaced by $-\boldsymbol{\alpha}_{-\rho} \boldsymbol{\alpha}_{+\rho}$. The latter means that in this bound state the electron and positron do not interact through the Coulomb potential and their retarded interaction occurs through the vector potential which is due to the particles transverse motion defined by the function $\varphi(\boldsymbol{\rho}, \boldsymbol{g})$.

As a result, for the massless composite boson state (9), (8) is reduced to

$$
\begin{aligned}
\varphi & \left(\boldsymbol{\rho}_{12}, \mathbf{g}\right) e^{i g z-i g t}=-i e^{2} \int_{-\infty}^{z} d z_{3} \int_{-\infty}^{z} d z_{4} \int d \boldsymbol{\rho}_{3} \int d \boldsymbol{\rho}_{4} \\
\cdot & \int_{-\infty}^{t} d t_{3} \int_{-\infty}^{t} d t_{4} \sum_{\mathbf{p} \boldsymbol{q}} \frac{e^{\left(i \mathbf{p}\left(\mathbf{r}_{1}-\mathbf{r}_{3}\right)+i \mathbf{q}\left(\mathbf{r}_{2}-\mathbf{r}_{4}\right)\right)}}{4 \varepsilon_{p} \varepsilon_{q}} \\
\cdot & \int_{-\infty}^{+\infty} e^{i|\omega| \rho_{34}} d \omega \\
\cdot & \left\{\Lambda_{-}^{+}(\mathbf{p}) e^{-i \varepsilon_{p}\left(t-t_{3}\right)}+\Lambda_{-}^{-}(\mathbf{p}) e^{i \varepsilon_{p}\left(t-t_{3}\right)}\right\} \\
\cdot & \left\{\Lambda_{+}^{+}(\mathbf{q}) e^{-i \varepsilon_{q}\left(t-t_{4}\right)}+\Lambda_{+}^{-}(\mathbf{q}) e^{i \varepsilon_{\mathbf{q}}\left(t-t_{4}\right)}\right\} \\
\cdot & \left\{e^{-i \omega\left(t_{3}-t_{4}\right)}+e^{-i \omega\left(t_{4}-t_{3}\right)}\right\} e^{i(g / 2)\left(z_{3}+z_{4}\right)-i(g / 2)\left(t_{3}+t_{4}\right)} \\
\cdot & \frac{1}{4 \pi \rho_{34}}\left(\boldsymbol{\alpha}_{-\rho} \boldsymbol{\alpha}_{+\rho}\right) \varphi\left(\boldsymbol{\rho}_{34}, \mathbf{g}\right)
\end{aligned}
$$

Here $\Lambda_{-}^{+}(\boldsymbol{p})=\varepsilon_{\boldsymbol{p}}+m \beta_{-}+\boldsymbol{\alpha}_{-} \boldsymbol{p}_{\rho}+p_{z}$ and $\Lambda_{-}^{-}(\boldsymbol{p})=\varepsilon_{\boldsymbol{p}}-$ $m \beta_{-}-\boldsymbol{\alpha}_{-} \boldsymbol{p}_{\rho}-p_{z}$ are the electron operators, $\Lambda_{+}^{+}(\boldsymbol{q})=\varepsilon_{\boldsymbol{q}}+$ $m \beta_{+}+\boldsymbol{\alpha}_{+} \boldsymbol{q}_{\rho}+q_{z}$ and $\Lambda_{+}^{-}(\boldsymbol{q})=\varepsilon_{\boldsymbol{q}}-m \beta_{+}-\boldsymbol{\alpha}_{+} \boldsymbol{q}_{\rho}-q_{z}$ are the positron ones, $m$ is the electron mass, $\varepsilon_{\boldsymbol{p}}=\sqrt{m^{2}+p^{2}}$ is the electron energy, and $\varepsilon_{\boldsymbol{q}}=\sqrt{m^{2}+q^{2}}$ is the positron energy. The matrices $\beta_{ \pm}$and $\boldsymbol{\alpha}_{ \pm}$are given in the standard representation.

At first, analyzing only the $z$ - dependent functions in (12), we integrate over $z_{3}$ and $z_{4}$ :

$$
\begin{aligned}
e^{i g z} & =\sum_{p_{z} q_{z}} \int_{-\infty}^{z \rightarrow \infty} d z_{3} \int_{-\infty}^{z \rightarrow \infty} d z_{4} \\
\cdot & e^{i(g / 2)\left(z_{3}+z_{4}\right)+i p_{z}\left(z-z_{3}\right)+i q_{z}\left(z-z_{4}\right)}=(2 \pi)^{2} \sum_{p_{z} q_{z}} e^{i\left(p_{z}+q_{z}\right) z} \\
& \cdot \delta\left(p_{z}-\frac{g}{2}\right) \delta\left(q_{z}-\frac{g}{2}\right)=\left.e^{i g z}\right|_{q_{z}=p_{z}=g / 2}
\end{aligned}
$$

Since the function $\varphi$ depends only on $\rho_{34}$, in (12), we replace the integration variables: $\int d \rho_{3} \int d \rho_{4}=$ $\int d \boldsymbol{\rho}_{34} \int d\left(\boldsymbol{\rho}_{3}+\boldsymbol{\rho}_{4}\right) / 2$. Thereafter the integral over $\left(\boldsymbol{\rho}_{3}+\boldsymbol{\rho}_{4}\right) / 2$ on the right side of (12) is easily calculated and gives us $(2 \pi)^{2} \delta\left(\boldsymbol{p}_{\rho}+\boldsymbol{q}_{\rho}\right)$. Consequently (12) takes the form:

$$
\begin{aligned}
\varphi\left(\boldsymbol{\rho}_{12}\right) e^{-i g t}=-i \frac{\alpha}{4 \pi} \int \frac{d \boldsymbol{\rho}_{34}}{\rho_{34}} \int_{-\infty}^{t} d t_{3} \int_{-\infty}^{t} d t_{4} \\
\cdot \sum_{\mathbf{p}_{\rho}} \frac{e^{i \mathbf{p}_{\rho}\left(\mathbf{r}_{12}-\mathbf{r}_{34}\right)}}{4 \varepsilon_{p}^{2}} \int_{-\infty}^{+\infty} e^{i|\omega| \rho_{34}} d \omega \\
\cdot\left\{\Lambda_{-}^{+}(\mathbf{p}) e^{-i \varepsilon_{p}\left(t-t_{3}\right)}+\Lambda_{-}^{-}(\mathbf{p}) e^{i \varepsilon_{p}\left(t-t_{3}\right)}\right\} \\
\cdot\left\{\Lambda_{+}^{+}(\mathbf{q}) e^{-i \varepsilon_{q}\left(t-t_{4}\right)}+\Lambda_{+}^{-}(\mathbf{q}) e^{i \varepsilon_{q}\left(t-t_{4}\right)}\right\} \\
\cdot\left\{e^{-i \omega\left(t_{3}-t_{4}\right)}+e^{-i \omega\left(t_{4}-t_{3}\right)}\right\} e^{-i(g / 2)\left(t_{3}+t_{4}\right)}\left(\boldsymbol{\alpha}_{-\rho} \boldsymbol{\alpha}_{+\rho}\right) \\
\cdot \varphi\left(\boldsymbol{\rho}_{34}, \mathbf{g}\right),
\end{aligned}
$$

where $\boldsymbol{q}_{\rho}=-\boldsymbol{p}_{\rho}, q_{z}=p_{z}=g / 2, \varepsilon_{p}=\varepsilon_{q}$, and $e^{2}$ was replaced by the fine structure constant, $\alpha$.

Now integrating over $t_{3}$ and $t_{4},(14)$ is rewritten as

$$
\begin{aligned}
\varphi\left(\boldsymbol{\rho}_{12}, \mathbf{g}\right)= & -i \frac{\alpha}{4 \pi} \int \frac{d \boldsymbol{\rho}_{34}}{\rho_{34}} \sum_{\mathbf{p}_{\rho}} \frac{e^{i \mathbf{p}_{\rho}\left(\rho_{12}-\rho_{34}\right)}}{4 \varepsilon_{p}^{2}} \\
& \cdot \int_{-\infty}^{\infty} e^{i|\omega| \rho_{34}} d \omega\left(I_{1}+I_{2}+I_{3}\right)\left(\boldsymbol{\alpha}_{-\rho} \boldsymbol{\alpha}_{+\rho}\right) \\
& \cdot \varphi\left(\boldsymbol{\rho}_{34}, \mathbf{g}\right),
\end{aligned}
$$

where the functions $I_{1,2,3}(\omega)$ are given by

$$
\begin{aligned}
I_{1}= & 2 \frac{\Lambda_{-}^{+}(\boldsymbol{p}) \Lambda_{+}^{+}(\boldsymbol{q})}{\omega^{2}-\left(\varepsilon_{p}-g / 2\right)^{2}+i \delta}, \\
I_{2} & =2 \frac{\Lambda_{-}^{-}(\boldsymbol{p}) \Lambda_{+}^{-}(\boldsymbol{q})}{\omega^{2}-\left(\varepsilon_{q}+g / 2\right)^{2}-i \delta}, \\
I_{3} & =\frac{\Lambda_{-}^{-}(\boldsymbol{p}) \Lambda_{+}^{+}(\boldsymbol{q})+\Lambda_{-}^{+}(\boldsymbol{p}) \Lambda_{+}^{-}(\boldsymbol{q})}{-g}\left(\frac{2 \varepsilon_{p}-g}{\omega^{2}-\left(\varepsilon_{p}-g / 2\right)^{2}+i \delta}\right. \\
& \left.+\frac{-2 \varepsilon_{p}-g}{\omega^{2}-\left(\varepsilon_{p}+g / 2\right)^{2}-i \delta}\right) .
\end{aligned}
$$

Here $\delta \rightarrow 0^{+}$defines the rule for bypassing simple poles.

All the three integrals over $\omega$ on the right side of (15) are easily calculated. We obtain

$$
\begin{aligned}
T_{1} & =\int_{-\infty}^{\infty} I_{1}(\omega) e^{i|\omega| \rho_{34}} d \omega \\
& =8 i \frac{\Lambda_{-}^{+}(\boldsymbol{p}) \Lambda_{+}^{+}(\boldsymbol{q})}{2 \varepsilon_{p}-g}(\cos (x) \operatorname{si}(x)-\sin (x) \operatorname{ci}(x)),
\end{aligned}
$$

where $x=\left|\varepsilon_{p}-g / 2\right| \rho_{34}$,

$$
\begin{aligned}
T_{2} & =\int_{-\infty}^{\infty} I_{2}(\omega) e^{i|\omega| \rho_{34}} d \omega=8 i \frac{\Lambda_{-}^{-}(\mathbf{p}) \Lambda_{+}^{-}(\mathbf{q})}{2 \varepsilon_{p}+g}\left\{\pi e^{i y}\right. \\
& +\cos (y) \operatorname{si}(y)-\sin (y) \operatorname{ci}(y)\}
\end{aligned}
$$


with $y=\left(\varepsilon_{p}+g / 2\right) \rho_{34}$ and

$$
\begin{aligned}
T_{3} & =\int_{-\infty}^{\infty} I_{3}(\omega) e^{i|\omega| \rho_{34}} d \omega=4 i \\
& \cdot \frac{\Lambda_{-}^{-}(\mathbf{p}) \Lambda_{+}^{+}(\mathbf{q})+\Lambda_{-}^{+}(\mathbf{p}) \Lambda_{+}^{-}(\mathbf{q})}{g}\left\{\pi e^{i y}\right. \\
& -\cos (x) \operatorname{si}(x)+\sin (x) \operatorname{ci}(x)+\cos (y) \operatorname{si}(y) \\
& -\sin (y) \operatorname{ci}(y)\} .
\end{aligned}
$$

Here $\operatorname{si}(x)$ and $\operatorname{ci}(x)$ are the integral sine and cosine functions.

Now we treat the bispinors of the function $\varphi$. It is apparent from (15) that the action of the following operators $\alpha_{-x} \alpha_{+x}+$ $\alpha_{-y} \alpha_{+y}, \Lambda_{-}^{+}(p) \Lambda_{+}^{+}(\boldsymbol{q}), \Lambda_{-}^{-}(p) \Lambda_{+}^{-}(\boldsymbol{q})$, and $\Lambda_{-}^{-}(p) \Lambda_{+}^{+}(q)+$ $\Lambda_{-}^{+}(p) \Lambda_{+}^{-}(q)$ on these bispinors must be reduced only to the multiplication of these spin functions on some scalars. For the boson state each particle of the bound pair can be characterized by the projection of the particle spin on the wave vector $\boldsymbol{g}$ or, in other words, the particle helicity. There are eight bispinor functions $\eta_{i}(i=1, \ldots, 8)$ for which the helicities of both the electron and positron are simultaneously either positive,

$$
\begin{gathered}
\eta_{i=1, \ldots, 4}=\left(\begin{array}{l}
1 \\
0 \\
0 \\
0
\end{array}\right)\left(\begin{array}{l}
1 \\
0 \\
0 \\
0
\end{array}\right),\left(\begin{array}{l}
1 \\
0 \\
0 \\
0
\end{array}\right)\left(\begin{array}{l}
0 \\
0 \\
1 \\
0
\end{array}\right), \\
\left(\begin{array}{l}
0 \\
0 \\
1 \\
0
\end{array}\right)\left(\begin{array}{l}
1 \\
0 \\
0 \\
0
\end{array}\right),\left(\begin{array}{l}
0 \\
0 \\
1 \\
0
\end{array}\right)\left(\begin{array}{l}
0 \\
0 \\
1 \\
0
\end{array}\right),
\end{gathered}
$$

or negative,

$$
\begin{gathered}
\eta_{i=5, \ldots, 8}=\left(\begin{array}{l}
0 \\
1 \\
0 \\
0
\end{array}\right)\left(\begin{array}{l}
0 \\
1 \\
0 \\
0
\end{array}\right),\left(\begin{array}{l}
0 \\
1 \\
0 \\
0
\end{array}\right)\left(\begin{array}{l}
0 \\
0 \\
0 \\
1
\end{array}\right), \\
\left(\begin{array}{l}
0 \\
0 \\
0 \\
1
\end{array}\right)\left(\begin{array}{l}
0 \\
1 \\
0 \\
0
\end{array}\right),\left(\begin{array}{l}
0 \\
0 \\
0 \\
1
\end{array}\right)\left(\begin{array}{l}
0 \\
0 \\
0 \\
1
\end{array}\right) .
\end{gathered}
$$

Because $\boldsymbol{\alpha}_{-\rho} \boldsymbol{\alpha}_{+\rho}=\alpha_{-x} \alpha_{+x}+\alpha_{-y} \alpha_{+y}$, one can verify that

$$
\boldsymbol{\alpha}_{-\rho} \boldsymbol{\alpha}_{+\rho} \eta_{i=1, \ldots, 8}=0
$$

It implies that the interaction function $G^{(1)}(3,4 ; 5,6) \propto$ $\boldsymbol{\alpha}_{-\rho} \boldsymbol{\alpha}_{+\rho}$ vanishes, and the massless boson state cannot be formed from these electron and positron states.
Analyzing the remaining possibilities, the required functions in (9) can be written as $\varphi_{1,2}=\chi_{1,2}(\boldsymbol{\rho}, \boldsymbol{g}) v_{1,2}$, where the bispinor functions are given by

$$
\begin{aligned}
& v_{1} \\
& =\frac{1}{\sqrt{2}}\left[\left(\begin{array}{l}
1 \\
0 \\
0 \\
0
\end{array}\right)\left(\begin{array}{l}
0 \\
0 \\
0 \\
1
\end{array}\right)+\left(\begin{array}{l}
0 \\
0 \\
0 \\
1
\end{array}\right)\left(\begin{array}{l}
1 \\
0 \\
0 \\
0
\end{array}\right)\right], \\
& \left.v_{2}=\frac{1}{\sqrt{2}}\left[\left(\begin{array}{l}
0 \\
1 \\
0 \\
0
\end{array}\right)\left(\begin{array}{l}
0 \\
0 \\
1 \\
0
\end{array}\right)+\left(\begin{array}{l}
0 \\
0 \\
1 \\
0
\end{array}\right){ }_{-}\left(\begin{array}{l}
0 \\
1 \\
0 \\
0
\end{array}\right)\right]+\right]
\end{aligned}
$$

and $\chi_{1,2}(\boldsymbol{\rho}, \boldsymbol{g})$ are the coordinate wave functions of the transverse motion of the strongly coupled electron-positron pair.

In the states (23) the helicities of the particles are opposite. For these functions we have

$$
\begin{aligned}
& \boldsymbol{\alpha}_{-\rho} \boldsymbol{\alpha}_{+\rho} \varphi_{1,2}=2 \varphi_{1,2}, \\
& \Lambda_{-}^{+}(\mathbf{p}) \Lambda_{+}^{+}(\mathbf{q}) \varphi_{1,2}=g\left(\varepsilon_{p}+\frac{g}{2}\right) \varphi_{1,2}, \\
& \Lambda_{-}^{-}(\mathbf{p}) \Lambda_{+}^{-}(\mathbf{q}) \varphi_{1,2}=g\left(-\varepsilon_{p}+\frac{g}{2}\right) \varphi_{1,2}, \\
& \left(\Lambda_{-}^{-}(\mathbf{p}) \Lambda_{+}^{+}(\mathbf{q})+\Lambda_{-}^{+}(\mathbf{p}) \Lambda_{+}^{-}(\mathbf{q})\right) \varphi_{1,2} \\
& \quad=\left(4 \varepsilon_{p}^{2}-g^{2}\right) \varphi_{1,2} .
\end{aligned}
$$

As a result, (15) is transformed to the following integral equation for the coordinate function $\chi_{1,2}$ (since $\chi_{1}=\chi_{2}$, the lower index 1, 2 can be omitted):

$$
\begin{aligned}
\chi\left(\boldsymbol{\rho}_{12}\right)= & \frac{\alpha}{(2 \pi)^{3}} \int \frac{d \boldsymbol{\rho}_{34}}{\rho_{34}} \int \frac{d \mathbf{p}_{\rho}}{\varepsilon_{p}^{2}} \exp \left(i \mathbf{p}_{\rho}\left(\boldsymbol{\rho}_{12}-\boldsymbol{\rho}_{34}\right)\right) \\
& \cdot\left(T_{1}+T_{2}+T_{3}\right) \chi\left(\boldsymbol{\rho}_{34}\right),
\end{aligned}
$$

where

$$
\begin{aligned}
T_{1} & =g \frac{2 \varepsilon_{p}+g}{2 \varepsilon_{p}-g}(\cos (x) \operatorname{si}(x)-\sin (x) \operatorname{ci}(x)), \\
T_{2} & =g \frac{g-2 \varepsilon_{p}}{g+2 \varepsilon_{p}}\left\{\pi e^{i y}+\cos (y) \operatorname{si}(y)-\sin (y) \operatorname{ci}(y)\right\}, \\
T_{3} & =\frac{4 \varepsilon_{p}^{2}-g^{2}}{g}\left\{\pi e^{i y}-\cos (x) \operatorname{si}(x)+\sin (x) \operatorname{ci}(x)\right. \\
& +\cos (y) \operatorname{si}(y)-\sin (y) \operatorname{ci}(y)\} .
\end{aligned}
$$


Equation (25) with the notations (26) is a rather complicated integral equation for the transverse wave function $\chi\left(\boldsymbol{\rho}_{12}, \boldsymbol{g}\right)$. It is important to show that this equation has solutions for the normalizable eigenfunctions. Below it will be demonstrated for the case of the $S$-state of the bound pair and small momentum of the boson, $g \ll m$. Then, $x=y=\varepsilon_{p} \rho_{34}$ can be used in (26).

For the $S$-state the angular momentum of the relative motion of the bound pair is zero, and the transverse wave function depends only on the modulus of the relative vector, that is, $\chi\left(\rho_{12}, g\right)$. In this case after integration over the azimuthal angle of the vector $\rho_{34}$ and integration over the azimuthal angle of the vector $\boldsymbol{p}_{\rho},(25)$ is reduced to

$$
\begin{aligned}
\chi\left(\rho_{12}\right)= & \frac{\alpha}{2 \pi} \int_{0}^{\infty} d \rho_{34} \int_{0}^{\infty} p_{\rho} d p_{\rho} J_{0}\left(p_{\rho} \rho_{12}\right) J_{0}\left(p_{\rho} \rho_{34}\right) \\
& \cdot \frac{\sum_{i=1}^{3} T_{i}\left(p, \rho_{34}\right)}{\varepsilon_{p}^{2}} \chi\left(\rho_{34}\right) .
\end{aligned}
$$

Here $J_{0}$ is the Bessel function of the first kind. In the case $g$ « $m$ from (26) we find that $T_{3} \gg T_{1}, T_{2}$ and

$$
T_{3} \cong 4 \pi \frac{\varepsilon_{p}^{2}}{g} \exp \left(i \varepsilon_{p} \rho_{34}\right) .
$$

Substituting (28) to (27), the latter equation is reduced to the homogeneous Fredholm integral equation of the second kind:

$$
\chi\left(\rho_{12}\right)=\frac{\alpha}{g} \int_{0}^{\infty} d \rho_{34} R\left(\rho_{12}, \rho_{34}\right) \chi\left(\rho_{34}\right)
$$

with the kernel

$$
\begin{aligned}
& R\left(\rho_{12}, \rho_{34}\right) \\
& \quad=2 \int_{0}^{\infty} p_{\rho} d p_{\rho} J_{0}\left(p_{\rho} \rho_{12}\right) J_{0}\left(p_{\rho} \rho_{34}\right) \exp \left(i \varepsilon_{p} \rho_{34}\right) .
\end{aligned}
$$

One can make sure that the kernel (30) is a non-Fredholm one. According to the asymptotic property of the Bessel function $J_{0}$, the integral in the right-hand side of (30) can only be defined as the principal value integral. That is, (29) with the kernel (30) is still difficult to solve.

In the momentum space, the equation for the transverse wave function can be reduced to a simpler form convenient for a numerical solution. To this end, in (25), we use the Fourier transform of the $\chi$-functions, $\chi(\boldsymbol{q})=$ $\int \chi\left(\boldsymbol{\rho}_{12}\right) \exp \left(-i \boldsymbol{q} \boldsymbol{\rho}_{12}\right) d \boldsymbol{\rho}_{12}$. Then, for the wave function in the momentum space we obtain:

$$
\begin{aligned}
& \chi(\mathbf{q}) \\
& \quad=\frac{\alpha}{(2 \pi)^{3}} \int \frac{d \boldsymbol{\rho}_{34}}{\rho_{34}} \frac{\sum_{i=1}^{3} T_{i}\left(q, \rho_{34}\right)}{\varepsilon_{q}^{2}} \int d \mathbf{f} e^{i(\mathbf{f}-\mathbf{q}) \boldsymbol{\rho}_{34}} \chi(\mathbf{f}) .
\end{aligned}
$$

For the $S$-state of the bound pair, (31) can be written as

$$
\begin{aligned}
\chi(q)= & \frac{\alpha}{2 \pi \varepsilon_{q}^{2}} \int d \rho_{34} \sum_{i=1}^{3} T_{i}\left(q, \rho_{34}\right) J_{0}\left(q \rho_{34}\right) \\
& \cdot \int f J_{0}\left(f \rho_{34}\right) \chi(f) d f .
\end{aligned}
$$

In the case $g \ll m$ the function $\sum_{i=1}^{3} T_{i}\left(q, \rho_{34}\right)$ can be replaced by the expression (28). Then (32) is reduced to the homogeneous Fredholm integral equation of the second kind:

$$
\chi(q)=\frac{\alpha}{g} \int_{0}^{\infty} Q(q, f) \chi(f) d f
$$

with the kernel

$$
\begin{aligned}
& Q(q, f) \\
& \quad=2 f \int_{0}^{\infty} J_{0}\left(q \rho_{34}\right) J_{0}\left(f \rho_{34}\right) \exp \left(i \varepsilon_{q} \rho_{34}\right) d \rho_{34} .
\end{aligned}
$$

Now the integral in the right-hand side of (34) that has a direct relationship with the discontinuous WeberSchafheitlin integral is an absolutely convergent integral which, however, is expressed through a discontinuous function, as will be shown below.

It is convenient to use the dimensionless variables: $x \rightarrow$ $\rho_{34} / \lambda_{e}, q \rightarrow q \lambda_{e}, f \rightarrow f \lambda_{e}$, and $g \rightarrow g m$. Here $\lambda_{e}=\hbar / m c$ is the Compton wavelength of the electron. Then (33) remains unchanged, and (34) is rewritten as

$$
\begin{aligned}
& Q(q, f) \\
& \quad=2 f \int_{0}^{\infty} J_{0}(q x) J_{0}(f x) \exp \left(i x \sqrt{1+q^{2}}\right) d x .
\end{aligned}
$$

The integral on the right-hand side of (35) was previously calculated [33]. Thus the complex $\operatorname{kernel} Q(q, f)=\operatorname{Re} Q+$ $i \operatorname{Im} Q$ is given by

$$
\operatorname{Im} Q= \begin{cases}\frac{4 f}{\pi \sqrt{1-f^{2}+2 f q}} \mathbf{K}\left(\frac{2 \sqrt{f q}}{\sqrt{1-f^{2}+2 f q}}\right), & f<\sqrt{1+q^{2}}-q \\ \frac{2}{\pi} \sqrt{\frac{f}{q}} \mathbf{K}\left(\frac{\sqrt{1-f^{2}+2 f q}}{2 \sqrt{f q}}\right), & \sqrt{1+q^{2}}-q<f<\sqrt{1+q^{2}}+q \\ 0, & f>\sqrt{1+q^{2}}+q,\end{cases}
$$




$$
\operatorname{Re} Q= \begin{cases}0, & f<\sqrt{1+q^{2}}-q \\ \frac{2}{\pi} \sqrt{\frac{f}{q} \mathbf{K}\left(\frac{\sqrt{f^{2}+2 f q-1}}{2 \sqrt{f q}}\right),} & \sqrt{1+q^{2}}-q<f<\sqrt{1+q^{2}}+q \\ \frac{4 f}{\pi \sqrt{f^{2}+2 f q-1}} \mathbf{K}\left(\frac{2 \sqrt{f q}}{\sqrt{f^{2}+2 f q-1}}\right), & f>\sqrt{1+q^{2}}+q\end{cases}
$$

Here $\boldsymbol{K}$ is the complete elliptic integral of the first kind. Since this kernel, having the weak logarithmic singularity, is a complex function, the boson wave function $\chi(q)$ is complex too. Partition of $\chi(q)$ into the imaginary and real parts is, in a sense, arbitrary since (33) is invariant under the phase transformation $\chi(q) \rightarrow \chi(q) e^{i \phi}$ with a constant $\phi$.

It is easy to see that the kernel (36)-(37) is a non-Fredholm one as well. Therefore we can expect that for this kernel the spectra of both the characteristic eigenvalues $g$ and the eigenfunctions $\chi(q, g)$ should be continuous.

2.3. Numerical Procedure. The studies of the Fredholm equation with non-Fredholm kernels are extremely rare in the literature because the search for its solutions is very difficult. In our case, the kernel eigenfunctions must satisfy the normalization condition, $2 \pi \int_{0}^{\infty}|\chi(q, g)|^{2} q d q=1$. Hence, $\chi(q \rightarrow \infty, g) \rightarrow 0$; that simplifies the problem. Assume that $\chi(q, g)$ goes to zero fast enough so that (33) can be reduced to the form:

$$
\chi(q)=\frac{\alpha}{g} \int_{0}^{f_{0}} Q(q, f) \chi(f) d f
$$

where the value of $f_{0}(g)$ in units of $\lambda_{e}^{-1}$ depends on the boson kinetic energy and $q \in\left[0, f_{0}\right]$.

The kernel $Q(q, f)$ given by (36)-(37) is replaced by the two $N \times N$ matrices:

$$
Q_{i j}^{(r)}+i Q_{i j}^{(i)}=\frac{f_{0}}{N-1} Q\left(q_{i}, f_{j}\right),
$$

which is denoted as $\mathbf{Q}^{(r)}+i \mathbf{Q}^{(i)}$. Here the number $N$ is the partition of the interval $\left[0, f_{0}\right]$. The wave function $\chi(q)$ is replaced by the two $N$-dimensional vectors $\chi(q) \Rightarrow \chi^{(r)}+$ $i \chi^{(i)}$. Then (38) is reduced to two related linear equations:

$$
\begin{aligned}
& \left(\frac{g}{\alpha} \mathbf{I}-\mathbf{Q}^{(r)}\right) \boldsymbol{\chi}^{(r)}=-\mathbf{Q}^{(i)} \boldsymbol{\chi}^{(i)}, \\
& \left(\frac{g}{\alpha} \mathbf{I}-\mathbf{Q}^{(r)}\right) \boldsymbol{\chi}^{(i)}=\mathbf{Q}^{(i)} \boldsymbol{\chi}^{(r)} .
\end{aligned}
$$

Here $\boldsymbol{I}$ is the unit $N \times N$ matrix.

From (40)-(41) we obtain the homogeneous system of linear $N$-equations for the vector $\chi^{(r)}$ :

$$
\mathbf{A} \chi^{(r)}=0
$$

where the $A$ matrix is

$$
\mathbf{A}=\frac{g}{\alpha} \mathbf{I}-\mathbf{Q}^{(r)}+\mathbf{Q}^{(i)}\left(\frac{g}{\alpha} \mathbf{I}-\mathbf{Q}^{(r)}\right)^{-1} \mathbf{Q}^{(i)}
$$

Here $\left((g / \alpha) \boldsymbol{I}-\mathbf{Q}^{(r)}\right)^{-1}$ means the inverse of $\left((g / \alpha) \boldsymbol{I}-\mathbf{Q}^{(r)}\right)$.
In contrast to the Fredholm procedure, to solve (42) with the definition (43), it is required to introduce a boundary condition. It is sufficient to put

$$
\operatorname{Re} \chi\left(f_{0}\right)=\delta \text {. }
$$

Here $\delta$ is small. Typically this value is assumed to be equal to $\delta=10^{-7}$. This selection does not matter because of the subsequent normalization of the wave function.

Using the boundary condition (44), from (42) we found the vector $\boldsymbol{\chi}^{(r)}$. Then, from (41) rewritten as

$$
\chi^{(i)}=\left(\frac{g}{\alpha} \mathbf{I}-\mathbf{Q}^{(r)}\right)^{-1} \mathbf{Q}^{(i)} \chi^{(r)}
$$

the vector $\chi^{(i)}$ was obtained. The function $\chi^{(r)}+i \chi^{(i)}$ was normalized to unity. Having thus determined the first approximation to the boson wave function $\chi(q),(40)$ was represented as

$$
\chi^{(r)}=-\left(\frac{g}{\alpha} \mathbf{I}-\mathbf{Q}^{(r)}\right)^{-1} \mathbf{Q}^{(i)} \chi^{(i)}
$$

and the system of two interrelated equations (45) and (46) was solved by the iterative method. The number of iterations necessary to provide a convergent solution appeared to be about a few hundred.

Then we found the average momentum of the transverse motion of the strongly coupled electron-positron pair:

$$
q_{a v} \lambda_{e}=2 \pi \int_{0}^{f_{0}}|\chi(q, g)|^{2} q^{2} d q
$$

The transverse wave function in the coordinate representation was derived $\left(x=\rho_{34} / \lambda_{e}\right)$ :

$$
\chi(x)=\int_{0}^{f_{0}} q J_{0}(x q) \chi(q) d q
$$

and the average transverse radius of the massless boson wave function was calculated:

$$
\frac{\rho_{a v}}{\lambda_{e}}=2 \pi \int_{0}^{f_{0}}|\chi(x, g)|^{2} x^{2} d x .
$$

In other words, $\rho_{a v}$ is the average relative distance between the electron and the positron in the massless boson state.

For all results presented below, $N=4501$ was used. To obtain reproducible results, the upper limit of integration $f_{0}$ (in units of $\lambda_{e}^{-1}$ ) has a bottom restriction which depends on the boson kinetic energy. Therefore, $f_{0}$ was chosen separately for each energy $g$. 


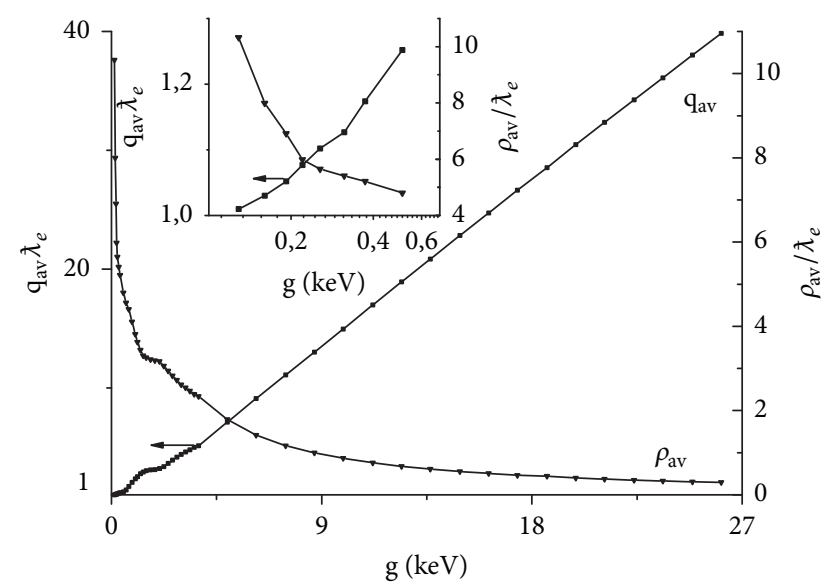

FIgURE 1: The average transverse momentum $q_{a v}$ (47) and the average transverse radius $\rho_{a v}$ (49) as functions of the massless boson kinetic energy. Inset: the same in the low-energy region.

2.4. Numerical Results for the Transverse Wave Functions. Figure 1 shows the kinetic energy dependencies of the integral characteristics (47) and (49) for the composite massless boson. With increasing energy $q_{a v}$ increases, and $\rho_{a v}$ decreases. In the low-energy region $129 \mathrm{eV} \leq g \leq 511 \mathrm{eV}$, with decreasing energy the average momentum $q_{a v}$ approaches monotonically to $\lambda_{e}^{-1}$, and the average transverse radius increases sharply, as shown in the inset in Figure 1. In a narrower region $129 \mathrm{eV} \leq g \leq 220 \mathrm{eV}$ the $g$ dependence of $\rho_{a v}$ is close to logarithmic, $\rho_{a v} \propto-\log (g)$. There is a reason to suppose that this logarithmic divergence persists to the limit $g \rightarrow 0$ because the massless particles cannot be at rest.

Because of computational constraints we were unable to carry out calculations for boson energies lower than $\simeq 130 \mathrm{eV}$. Figure 2 demonstrates the boson wave function for the energy $g=160 \mathrm{eV}$ (the dimensionless value of $g=0.043 \alpha$ ) that is slightly larger than the boson energy restriction. Above we pointed out the arbitrariness of the choice between the imaginary and real parts of $\chi$. Therefore we do not introduce the corresponding notations for the presented curves. Note that Figure 2 shows one of the possible representations of $\chi(q)$ obtained in our calculations, since the phase transformation of the wave function will change these curves.

The momentum wave function is maximal at $q=0$ and then abruptly decays with increasing $q$. This feature for small boson energies does not allow us to calculate accurately the coordinate wave function at $\rho=0$, since the integrand function in (48) vanishes at $q=0$. However, this does not affect the average transverse radius of the massless boson wave function. It was found that $q_{a v}=1.03 \lambda_{e}^{-1}$ and $\rho_{a v}=$ $7.99 \lambda_{e}$ for the energy $g=160 \mathrm{eV}$.

Computational noise on the curves presented in Figure 2(a) correlates with the step of the finite difference grid $\Delta q=f_{0}(g) /(N-1)$. With decreasing energy $g<160 \mathrm{eV}$ the region of this noise becomes more extended. After the transformation (48) the noise features vanish, as shown in Figure 2(b).
According to Figure 1, with increasing boson energy, there is the extension of the transverse wave function in the momentum space, and the contraction of the real space coordinate wave function. This contraction means that the electron and the positron become closer to each other in the $\boldsymbol{\rho}$-space. The region $1 \mathrm{keV}<g<2.3 \mathrm{keV}$ can be considered as a transition region, in which $q_{a v} \lambda_{e} \simeq \rho_{a v} \lambda_{e}^{-1}$. Outside this region $\rho_{a v}$ decreases monotonically with increasing boson energy, and the dependence of $q_{a v}(g)$ becomes close to linear. Figure 3 shows both the momentum and coordinate wave functions for $g=1.68 \mathrm{keV}$.

Comparing the data in Figures 2 and 3, one can conclude that, with increasing of the boson energy, oscillations of the wave functions in both the momentum and coordinate spaces are enhanced. The wave function in the momentum space presented in Figure 3(a) is more extended as compared with that for $g=160 \mathrm{eV}$ (see Figure 2(a)). Consequently, the average transverse momentum increases to the value of $q_{a v}=3.09 \lambda_{e}^{-1}$. The probability of zero relative momentum of the particles decreased significantly in comparison to that presented in Figure 2(a). As a consequence of the contraction of the transverse wave function, the average transverse radius of the boson wave function is changed from $\rho_{a v}=7.99 \lambda_{e}$ at $g=160 \mathrm{eV}$ (Figure 2(b)) to the value $\rho_{a v}=3.22 \lambda_{e}$ at $g=1.68 \mathrm{keV}$ (Figure 3(b)).

With a further increase in the boson energy the transverse contraction of the wave function $\chi(\rho, g)$ tends to slow down. At the same time the probability of finding the electron and positron with nearly zero distance between them increases abruptly. This is clearly demonstrated by Figure 4 where the boson state corresponding to the kinetic energy $g=24.87 \mathrm{keV}$ is presented. The transverse momentum wave function (Figure $4(\mathrm{a})$ ) is very extended, and the average relative transverse momentum $q_{a v}=38.02 \lambda_{e}^{-1}$. The wave function in coordinate space is presented in Figure $4(\mathrm{~b})$. The average transverse radius between the electron and positron $\rho_{a v}=0.31 \lambda_{e}$, but with the highest probability density the relative distance between the particles $\rho<0.1 \lambda_{e}$.

Thus, the consideration of the electron and positron as independent particles leads to the appearance of the branch of the massless composite bosons formed by the coupled electron-positron pairs with the coupling equal to the fine structure constant. The results obtained above for the normalizable wave functions of the massless bosons are used in the next section, in which reaction (1) is investigated theoretically.

\section{The $2 \gamma$ Angular Correlation Spectrum}

3.1. The Initial and Final States of the Reaction (1). We will need the massless boson state for an arbitrary direction of the boson momentum. When $\boldsymbol{g}$ is directed along the $z$ axis, the boson wave function is given by (9) with $\varphi_{1,2}=$ $\chi(\boldsymbol{\rho}, \boldsymbol{g}) v_{1,2}$, where the bispinor parts $v_{1,2}$ have the forms $(23)$. The bispinors $v_{1,2}$ define only the $z$-projection of the total spin $\left(\Sigma_{z-}+\Sigma_{z+}\right) v_{1,2}=0$. That is, the helicities of the particles are opposite. 


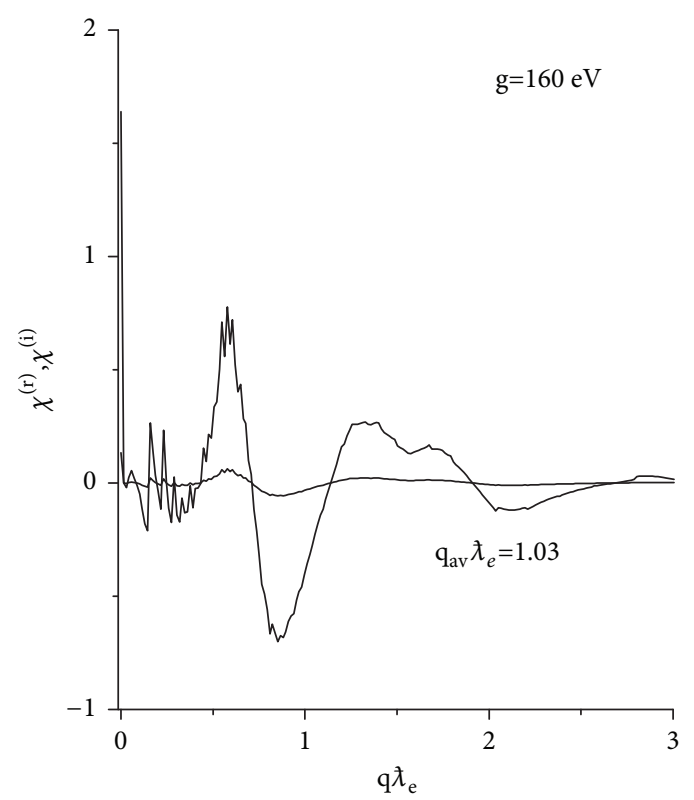

(a)

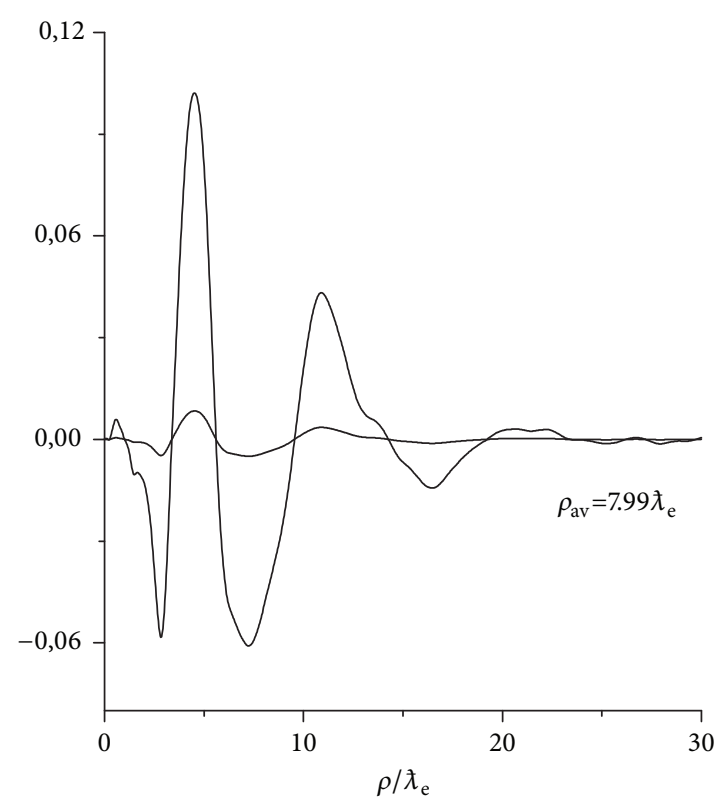

(b)

FIGURE 2: The momentum wave function (a) and the coordinate wave function (b) for the massless boson energy $160 \mathrm{eV}$. The parameter $f_{0}=65$.

When $\boldsymbol{g}$ is directed along the $x$-axis, the bispinors take the forms:

$$
\begin{aligned}
& v_{1}=\frac{1}{\sqrt{8}}\left[\left(\begin{array}{l}
1 \\
1 \\
0 \\
0
\end{array}\right)\left(\begin{array}{c}
0 \\
0 \\
1 \\
-1
\end{array}\right)+\right. \\
& \left.+\left(\begin{array}{c}
0 \\
0 \\
1 \\
-1
\end{array}\right)\left(\begin{array}{l}
1 \\
1 \\
0 \\
0
\end{array}\right)\right] \\
& v_{2}=\frac{1}{\sqrt{8}}\left[\left(\begin{array}{c}
1 \\
-1 \\
0 \\
0
\end{array}\right)\left(\begin{array}{l}
0 \\
0 \\
1 \\
1
\end{array}\right)+\right. \\
& \left.+\left(\begin{array}{l}
0 \\
0 \\
1 \\
1
\end{array}\right)\left(\begin{array}{c}
1 \\
-1 \\
0 \\
0
\end{array}\right)\right]
\end{aligned}
$$

For (50) we have $\left(\Sigma_{x_{-}}+\Sigma_{x_{+}}\right) v_{1,2}=0$. The form of these functions is obvious when $\boldsymbol{g}$ is parallel to the $y$-axis.

Now consider an arbitrary direction of the momentum boson. Let $\boldsymbol{r}_{\perp}$ be an arbitrary radius vector lying in the plane perpendicular to the vector $\boldsymbol{g}\left(\boldsymbol{g} \boldsymbol{r}_{\perp}=0\right)$. Then the wave function (9) takes the form:

$$
\psi_{1,2}=\chi\left(\mathbf{r}_{\perp}, g\right) v_{1,2}\left(\mathbf{i}_{\mathbf{g}}\right) \exp \left(i \mathbf{g} \boldsymbol{r}_{g}-i g t\right)
$$

where $\boldsymbol{r}_{g}=\boldsymbol{r}-\boldsymbol{r}_{\perp}$ is the component of the radius vector $\boldsymbol{r}$ which is collinear to $\boldsymbol{g}$ and $v_{1,2}$ are the bispinor parts which can depend only on the angles of the unit vector $\boldsymbol{i}_{\boldsymbol{g}}=\boldsymbol{g} / \boldsymbol{g}$. For the wave function (51) one should understand that $\boldsymbol{r}_{\perp}=\boldsymbol{r}_{1 \perp}-\boldsymbol{r}_{2 \perp}$, where $\boldsymbol{g} \boldsymbol{r}_{1 \perp}=0$ and $\boldsymbol{g} \boldsymbol{r}_{2 \perp}=0$, and that $\boldsymbol{r}_{1 g}=\boldsymbol{r}_{1}-\boldsymbol{r}_{1 \perp}$ and $\boldsymbol{r}_{2 g}=\boldsymbol{r}_{2}-\boldsymbol{r}_{2 \perp}$ with the condition $\boldsymbol{r}_{1 g}=\boldsymbol{r}_{2 g}=\boldsymbol{r}_{g}$.

As it is well known, the three-dimensional spinors corresponding to definite helicities have the following form:

$$
\begin{gathered}
w_{1 / 2}=\left(\begin{array}{c}
e^{-i \varphi_{g} / 2} \cos \frac{\theta_{g}}{2} \\
e^{i \varphi_{g} / 2} \sin \frac{\theta_{g}}{2}
\end{array}\right), \\
w_{-1 / 2}=\left(\begin{array}{c}
-e^{-i \varphi_{g} / 2} \sin \frac{\theta_{g}}{2} \\
e^{i \varphi_{g} / 2} \cos \frac{\theta_{g}}{2}
\end{array}\right),
\end{gathered}
$$

where $\theta_{g}$ and $\varphi_{g}$ are the polar and azimuthal angles of the vector $\boldsymbol{i}_{\boldsymbol{g}}$. Taking into account (52), one can see that (23) and (50) are the special cases of the following bispinor functions: 


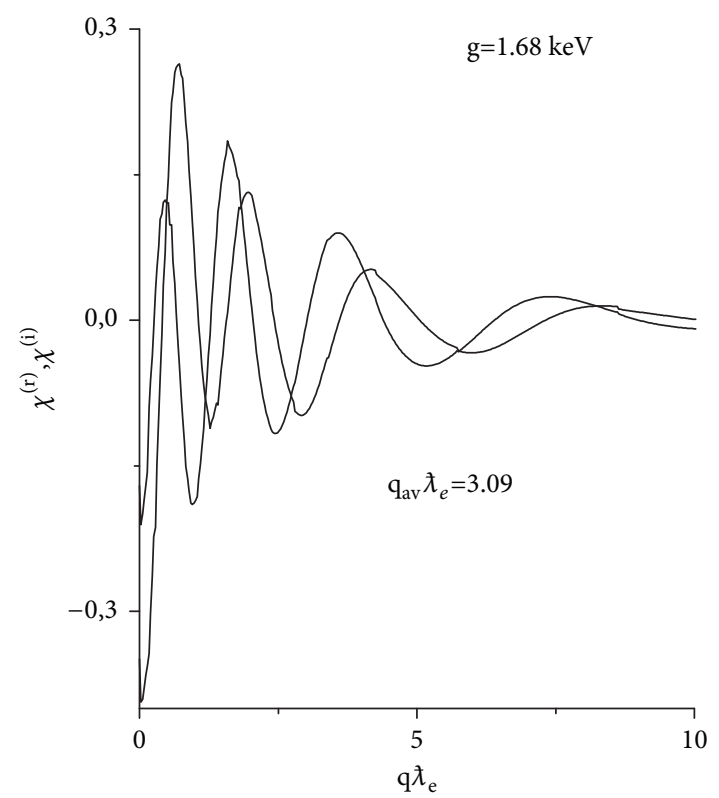

(a)

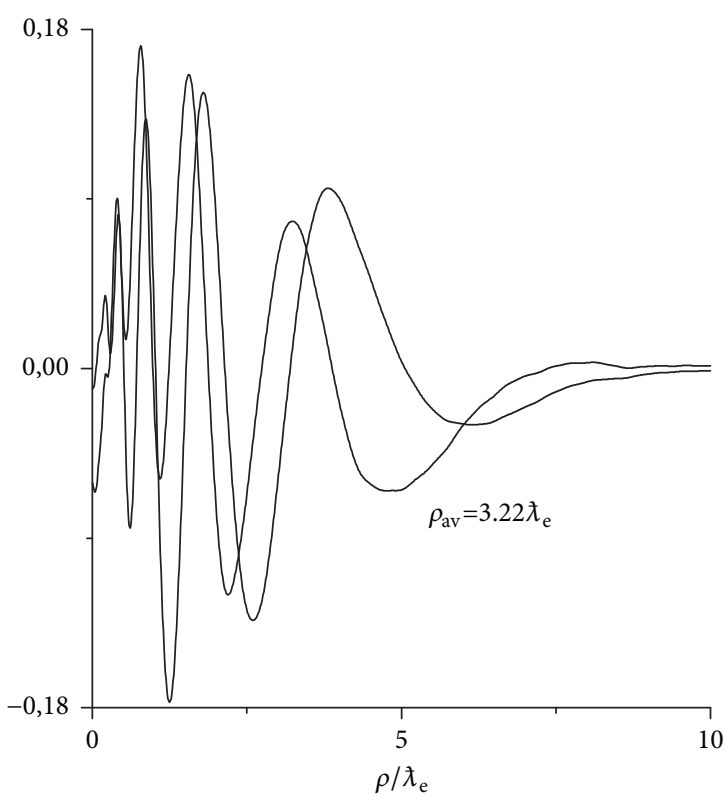

(b)

FIgURE 3: The same as in Figure 2 except for the massless boson energy equal to $1.68 \mathrm{keV}$. The parameter $f_{0}=130$.

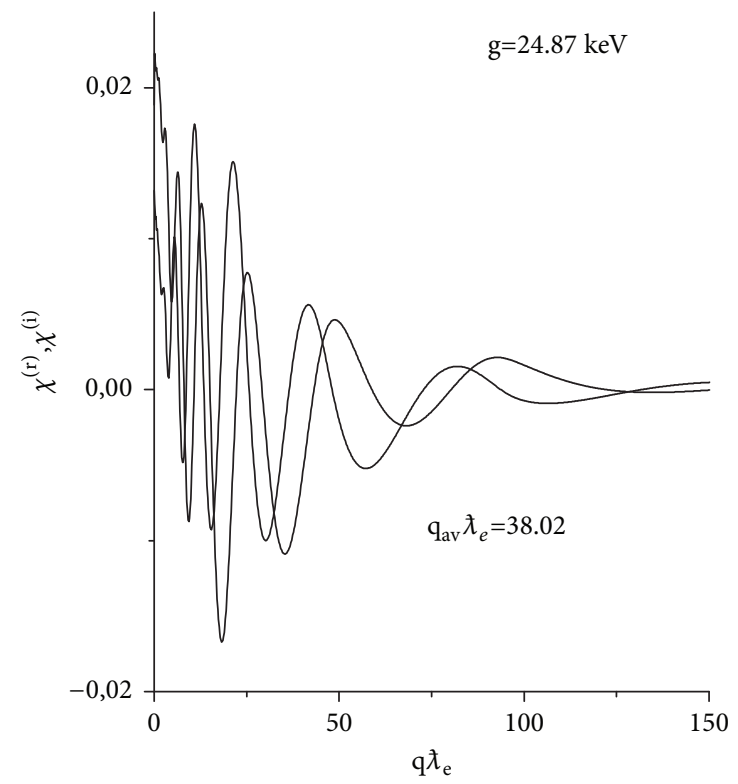

(a)

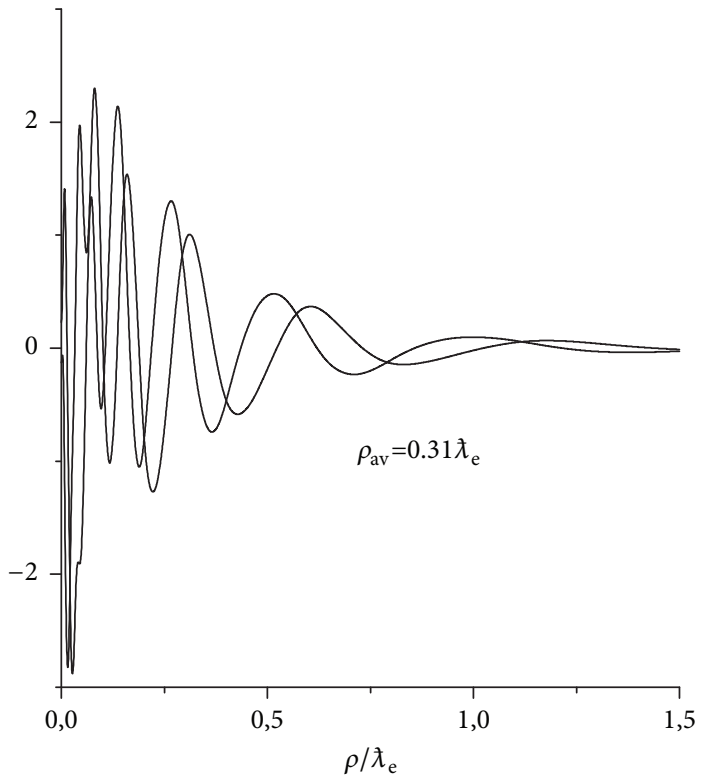

(b)

Figure 4: The massless boson wave function in the momentum space (a) and the coordinate space (b) for the kinetic energy $24.87 \mathrm{keV}$. The parameter $f_{0}=767$.

$$
\begin{aligned}
& v_{1} \\
& =\frac{1}{\sqrt{2}}\left[\left(\begin{array}{c}
w_{1 / 2} \\
0
\end{array}\right)_{-}\left(\begin{array}{c}
0 \\
w_{-1 / 2}
\end{array}\right)_{+}+\left(\begin{array}{c}
0 \\
w_{-1 / 2}
\end{array}\right)_{-}\left(\begin{array}{c}
w_{1 / 2} \\
0
\end{array}\right)_{+}\right], \\
& v_{2} \\
& =\frac{1}{\sqrt{2}}\left[\left(\begin{array}{c}
w_{-1 / 2} \\
0
\end{array}\right)_{-}\left(\begin{array}{c}
0 \\
w_{1 / 2}
\end{array}\right)_{+}+\left(\begin{array}{c}
0 \\
w_{1 / 2}
\end{array}\right)_{-}\left(\begin{array}{c}
w_{-1 / 2} \\
0
\end{array}\right)_{+}\right] .
\end{aligned}
$$

Now we turn to the initial state of the free electron and positron. In principle one can consider colliding non-spinpolarized particles with equal energy. Currently, however, the production of spin-polarized low-energy positron beams with the kinetic energy of a few electron volts became possible [34-38]. It is therefore of particular interest to study the annihilation-like process (1) for the polarized beams.

Suppose that the electron and positron collide with the center of mass at rest $\left(\boldsymbol{p}_{-}+\boldsymbol{p}_{+}=0\right)$. For definiteness let the 
electron momentum, $\boldsymbol{p}_{-}$, be directed along the $z$-axis; then the positron momentum, $\boldsymbol{p}_{+}$, is against the $z$-axis. The wave function of the free pair can be represented as

$$
\varphi(1,2)=u \exp \left(-i p_{-} x_{-}-i p_{+} x_{+}\right),
$$

where $p_{-}=\left(\varepsilon_{-}, p_{-}\right)$and $x_{-}=(t, r)$ are the four vectors for the electron, $p_{+}=\left(\varepsilon_{+}, \boldsymbol{p}_{+}\right)$and $x_{+}=\left(t, \boldsymbol{r}_{1}\right)$ are the four vectors of the positron, and $\varepsilon_{-}=\varepsilon_{+}=\varepsilon$. The spin function in (54) can be defined by the $z$-projections of the spin for the particles in the rest system. Then, for the total spin projection $S_{z}=0$, the function $u$ can be represented as

$$
u_{S_{z}=0}=\left(\begin{array}{c}
\sqrt{\varepsilon+m} \\
0 \\
\sqrt{\varepsilon-m} \\
0
\end{array}\right)\left(\begin{array}{c}
0 \\
\sqrt{\varepsilon+m} \\
0 \\
\sqrt{\varepsilon-m}
\end{array}\right)_{+}
$$

The function (55) can be considered as the two-particle spin state with opposite helicities. One can also say that (55) corresponds to the opposite longitudinal polarization of the particles.

Free pairs of the transversely polarized particles relative to the vector $\boldsymbol{p}_{-}$can be prepared as well, and it is easy to write, for example, $u_{S_{x}=0}$ for the polarization along the $x$-axis. Besides, the colliding electron-positron pair with $S_{z}=1$ or $S_{x}=1$ can be experimentally obtained, and it is not difficult to write the corresponding bispinor parts for these cases.

Interaction for the reaction (1), which will be discussed in Section 3.2, predetermines the choice of the photon wave function. The photon plane wave can be represented as

$$
A_{k}^{(\alpha)}=\sqrt{4 \pi} \mathbf{l}_{\mathbf{k}}^{(\alpha)} \exp (-i k x),
$$

where $\boldsymbol{l}_{\boldsymbol{k}}^{(\alpha)}$ is the photon polarization that can be chosen real, $k x=\omega_{k} t-\boldsymbol{k} \boldsymbol{r}$, and $\omega_{k}, \boldsymbol{k}$ are the energy and wave vector of the photon, respectively. For the transverse photons $\boldsymbol{k} \boldsymbol{l}_{k}^{(\alpha)}=0$ with $\alpha=1,2$.

3.2. Interaction for the Reaction (1). The products of reaction (1) include two photons and the massless boson. This composite boson is formed by the strongly coupled electronpositron pair. Therefore, the radiative transition of any particle from the initial free pair (the left-hand side of (1)) to any intermediate state does not lead to the formation of the massless boson. The simultaneous emission of one photon by the electron and other photon by the positron and, accordingly, the simultaneous transition of both the electron and positron into the massless boson state are the only processes for which reaction (1) occurs. For this reason it is necessary to determine the interaction operator for the simultaneous emission of photons by each particle of the pair.

The additive energy of any free pair from the beams is defined as

$$
\varepsilon_{-}+\varepsilon_{+}=\sqrt{m_{s}^{2}+\left(\mathbf{p}_{-}+\mathbf{p}_{+}\right)^{2}}
$$

Here $m_{s}$ is the pair mass. Note that in (57) the sum of the particle momenta is presented.
The interaction operator for single-photon emission by the electron and positron at the same time can be obtained from (57) by introducing the canonical momenta in the presence of electromagnetic fields, $\boldsymbol{p}_{-} \rightarrow \boldsymbol{p}_{-}+e \boldsymbol{A}_{-}$and $\boldsymbol{p}_{+} \rightarrow \boldsymbol{p}_{+}-e \boldsymbol{A}_{+}\left(\boldsymbol{A}_{ \pm}\right.$are the operators of the vector potentials generated by the electron and positron). Assuming $m_{s}=$ $2 m$ for the nonrelativistic particles, from (57), we obtain the expression for this operator:

$$
W=-\frac{e^{2}}{2 m} \mathbf{A}_{-} \mathbf{A}_{+} .
$$

A similar approach can be applied to positronium. The kinetic energy of the unperturbed Hamiltonian is

$$
T=\frac{\mathbf{p}^{2}}{m},
$$

where $\boldsymbol{p}$ is the momentum operator of the relative motion, corresponding to the relative radius vector between the particles. Substituting $\boldsymbol{p}=\left(\boldsymbol{p}_{-}-\boldsymbol{p}_{+}\right) / 2$ into this energy and making the transition to the canonical momenta of the particles, we get, up to the sign, the operator (58) for simultaneous emission of two photons by the two particles.

The electromagnetic interaction operator (58) is used below in the study of the reaction (1).

3.3. Possible Reaction Channels. Now, taking into account the spin conservation, we discuss the possible reaction channels with initially free electron-positron pairs. Let the colliding pairs be in the triplet state, $S_{z}=1$. First of all, the reaction with single-photon emission

$$
\left(e^{-} e^{+}\right)_{S_{z}=1} \longrightarrow B \gamma
$$

is impossible in principle. Here $\left(e^{-}+e^{+}\right)_{S_{z}=1}$ denotes the initial free pair, $B$ is the massless boson, and $\gamma$ is the photon emitted. For this reaction, the single photon is only emitted either by the electron or by the positron. Therefore, the simultaneous transition of these particles into the composite massless boson state cannot occur.

The reaction with emission of three photons,

$$
\left(e^{-} e^{+}\right)_{S_{z}=1} \longrightarrow B \gamma_{1} \gamma_{2} \gamma_{3}
$$

can take place. For (61), at first one of the particles (electron or positron) emits one photon ( say $\gamma_{1}$ ) and passes to an intermediate state, and then, in a subsequent point in time, simultaneous emission of two other photons $\left(\gamma_{2}, \gamma_{3}\right)$ is accompanied by the simultaneous transition of these particles into the boson state $B$.

In the case of prepared pairs with $S_{z}=0$ the reaction with single-photon emission is also impossible in principle as was discussed above.

The reaction with the emission of two photons:

$$
\left(e^{-} e^{+}\right)_{S_{z}=0} \longrightarrow B \gamma_{1} \gamma_{2}
$$

can occur due to the interaction (58). As will be shown below, for (62), the emission of composite bosons with relatively low 
energies is possible and preferable. Their energy is several orders of magnitude smaller than the electron mass. With the increasing of the boson energy the cross-section of this reaction decreases sharply.

Below, we derive the cross-section of the reaction (62) and study numerically the $2 \gamma$ angular correlation spectra.

3.4. Cross-Section of the Reaction (62). In the center of mass system $\boldsymbol{p}_{-}=-\boldsymbol{p}_{+}=\boldsymbol{p}$ and $\varepsilon_{-}+\varepsilon_{+}=2 \varepsilon$. The cross-section of the reaction (62) has the following form:

$$
\begin{aligned}
d \sigma= & (2 \pi)^{4} \delta^{(4)}\left(P_{i}-P_{f}\right)\left|W_{i f}\right|^{2} \frac{1}{4 \varepsilon^{2} v_{\text {rel }}} \frac{d \mathbf{k}}{(2 \pi)^{3} 2 \omega} \\
& \cdot \frac{d \mathbf{k}_{1}}{(2 \pi)^{3} 2 \omega_{1}} \frac{d \mathbf{g}}{(2 \pi)^{3} 2 g} .
\end{aligned}
$$

Here $\delta^{(4)}\left(P_{i}-P_{f}\right)=\delta\left(\omega+\omega_{1}+g-2 \varepsilon\right) \delta\left(\boldsymbol{k}+\boldsymbol{k}_{1}+\boldsymbol{g}\right)$, $v_{\text {rel }}=2 v_{e}$ is the relative velocity of the particles, $v_{e}=$ $\sqrt{2(\varepsilon-m) / m}$, and $W_{i f}$ is the matrix element of the operator (58) for the transition from the initial state $(i)$ of the free pair (54)-(55) into the final state $(f)$. The latter include the composite massless boson (51)-(53) and the two photons (56), one of which has the energy $\omega$, the momentum $\boldsymbol{k}$, and the polarization $\boldsymbol{l}_{\boldsymbol{k}}^{(\alpha)}(\alpha=1,2)$ and the second photon, $\omega_{1}, \boldsymbol{k}_{1}$, and $\boldsymbol{l}_{\boldsymbol{k}_{1}}^{(\beta)}(\beta=1,2)$, respectively. This matrix element is written as

$$
\begin{gathered}
W_{i f}=-4 \pi \frac{e^{2}}{2 m}\left(\mathbf{l}_{\mathbf{k}}^{(\alpha)} \mathbf{l}_{\mathbf{k}_{1}}^{(\beta)}\right)\left(v_{1,2}^{+} u_{S_{z}=0}\right) \int d \mathbf{r}_{1} \int d \mathbf{r}_{2} \\
\chi^{*}\left(\left|\mathbf{r}_{\perp}\right| ; g\right) \times e^{-i \mathbf{k} \boldsymbol{r}_{1}-i \mathbf{k}_{1} \mathbf{r}_{2}-i \mathbf{g} \boldsymbol{r}_{g}}\left(e^{i \mathbf{p} \boldsymbol{r}_{1}-i \mathbf{p} \boldsymbol{r}_{2}}+e^{i \mathbf{p} \boldsymbol{r}_{2}-i \mathbf{p} \boldsymbol{r}_{1}}\right) .
\end{gathered}
$$

Here $\chi\left(\left|\boldsymbol{r}_{\perp}\right| ; g\right)$ satisfies (29), $\boldsymbol{r}_{\perp}=\boldsymbol{r}_{1 \perp}-\boldsymbol{r}_{2 \perp}$ is the two-dimensional relative vector between the electron and positron, $\boldsymbol{r}_{1}=\boldsymbol{r}_{1 \perp}+\boldsymbol{r}_{1 g}$, and $\boldsymbol{r}_{2}=\boldsymbol{r}_{2 \perp}+\boldsymbol{r}_{2 g}$. As noted above, when the boson momentum is directed along $z$-axis, the $z$ components of the radius-vectors of particles coincide, and $z_{1}=z_{2}=z$. Now we need to take into account the fact that $r_{1 g}=r_{2 g}=r_{g}$ for the composite boson. On the right side of (64), the last factor in round brackets is the sum of two terms. The first contribution corresponds to the emission of photon with the wave vector $\boldsymbol{k}$ by the electron, and in the second term the electron emits photon with the wave vector $\boldsymbol{k}_{1}$. The second of two photons is emitted simultaneously by the positron.

To calculate the spatial integrals in (64), it is convenient to use new variables: $\boldsymbol{r}_{1 \perp}=\boldsymbol{R}+\boldsymbol{r}_{\perp} / 2$ and $\boldsymbol{r}_{2 \perp}=\boldsymbol{R}-\boldsymbol{r}_{\perp} / 2$. As a result we obtain

$$
\begin{aligned}
& \int d \mathbf{r}_{1} \int d \mathbf{r}_{2} \chi^{*}\left(\left|\mathbf{r}_{1 \perp}-\mathbf{r}_{2 \perp}\right| ; g\right) \\
& \cdot e^{-i \mathbf{k} \boldsymbol{r}_{1}-i \mathbf{k}_{1} \mathbf{r}_{2}-i \mathbf{g} \boldsymbol{r}_{g}}\left(e^{i \mathbf{p} \boldsymbol{r}_{1}-i \mathbf{p} \boldsymbol{r}_{2}}+e^{i \mathbf{p} \boldsymbol{r}_{2}-i \mathbf{p} \mathbf{r}_{1}}\right) \\
& =\chi^{*}\left(\left|\mathbf{k}_{\perp}-\mathbf{p}_{\perp}\right| ; g\right)+\chi^{*}\left(\left|\mathbf{k}_{\perp}+\mathbf{p}_{\perp}\right| ; g\right)
\end{aligned}
$$

multiplied by $(2 \pi)^{3} \delta\left(\boldsymbol{k}+\boldsymbol{k}_{1}+\boldsymbol{g}\right)$. We do not write the latter, because it is already included in (63). In (65) the Fourier transform of the wave function of the transverse motion of the coupled pair in the massless composite boson state is used:

$$
\chi^{*}\left(\left|\mathbf{k}_{\perp}+\mathbf{p}_{\perp}\right| ; g\right)=\int \chi^{*}\left(\left|\mathbf{r}_{\perp}\right| ; g\right) e^{-i(\mathbf{k}+\mathbf{p}) \mathbf{r}_{\perp}} d \mathbf{r}_{\perp} .
$$

Here $\boldsymbol{k}_{\perp}$ is the component of the photon wave vector $\boldsymbol{k}$ which is perpendicular to $\boldsymbol{g}$,

$$
\mathbf{k}_{\perp}=\mathbf{k}-\left(\mathbf{k} i_{\mathrm{g}}\right) \mathbf{i}_{\mathrm{g}},
$$

and $\boldsymbol{p}_{\perp}$ is the component of the free electron momentum perpendicular to the boson momentum,

$$
\mathbf{p}_{\perp}=\mathbf{p}-\left(\mathrm{p} i_{\mathrm{g}}\right) \mathbf{i}_{\mathrm{g}} .
$$

According to the momentum conservation, $\boldsymbol{k}_{\perp}+\boldsymbol{k}_{1 \perp}=0$, where $\boldsymbol{k}_{1 \perp}$ is the corresponding component of the wave vector of the second emitted photon.

Note that $\chi^{*}\left(\left|\boldsymbol{k}_{\perp} \pm \boldsymbol{p}_{\perp}\right| ; g\right)$ has the dimension of length.

Substituting (64)-(65) in (63), the cross-section is given by

$$
\begin{aligned}
d \sigma= & 2^{-8} \pi^{-3} r_{e}^{2} \frac{c}{v_{\text {rel }}} \sum_{\alpha, \beta}\left|\mathbf{l}_{\mathbf{k}}^{(\alpha)} \mathbf{l}_{\mathbf{k}_{1}}^{(\beta)}\right|^{2} \frac{1}{\varepsilon^{2}}\left|v_{1,2}^{+} u_{S_{z}=0}\right|^{2} \\
\cdot & \left|\chi\left(\left|\mathbf{k}_{\perp}-\mathbf{p}_{\perp}\right| ; g\right)+\chi\left(\left|\mathbf{k}_{\perp}+\mathbf{p}_{\perp}\right| ; g\right)\right|^{2} \\
& * \delta\left(k+k_{1}+g-2 k_{e}\right) \\
& \cdot \delta\left(\mathbf{k}_{1}+\mathbf{k}+\mathbf{g}\right) \frac{d \mathbf{k}}{k} \frac{d \mathbf{k}_{1}}{k_{1}} \frac{d \mathbf{g}}{g}
\end{aligned}
$$

where $r_{e}=e^{2} / m$ is the electromagnetic radius of the electron and $k_{e}=\lambda_{e}^{-1} * \varepsilon / m$, where $\lambda_{e}$ is the Compton wavelength of the electron.

3.5. Transformation of (69). In (69) we have the summation over photon polarization: $S=\sum_{\alpha, \beta}\left|\boldsymbol{l}_{\boldsymbol{k}}^{(\alpha)} \boldsymbol{l}_{\boldsymbol{k}_{1}}^{(\beta)}\right|^{2}$. Because $l_{\boldsymbol{k} i}^{(\alpha)} l_{\boldsymbol{k} j}^{(\alpha)}=0$ for $i, j=x, y, z$ and $i \neq j$, the sum is reduced to

$$
S=\sum_{\alpha, \beta i=x, y, z} \sum_{\mathbf{k} i}^{(\alpha)^{2}} l_{\mathbf{k}_{1} i}^{(\beta)^{2}} .
$$

For the unit polarization vectors we can use $l_{\boldsymbol{k} z}^{(\alpha)^{2}}=1-l_{\boldsymbol{k} x}^{(\alpha)^{2}}-$ $l_{\boldsymbol{k} y}^{(\alpha)^{2}}$. Then, taking into account $\sum_{\alpha=1,2} l_{k x}^{(\alpha)^{2}}=1$, finally we obtain $S=2$.

In (69) the factor $\left|v_{1,2}^{+} u_{S_{z}=0}\right|^{2}$ can be considered as the overlap of bispinor functions. Using (53) and (55), we find that the factor is equal to $(1 / 2) p^{2} \cos ^{2} \theta_{g}$.

Considering these results, in the nonrelativistic limit, the cross-section (69) is reduced to

$$
\begin{aligned}
d \sigma= & 2^{-9} \pi^{-3} r_{e}^{2} \frac{v_{e}}{c} \cos ^{2} \theta_{g} \\
& \cdot\left|\chi\left(\left|\mathbf{k}_{\perp}-\mathbf{p}_{\perp}\right| ; g\right)+\chi\left(\left|\mathbf{k}_{\perp}+\mathbf{p}_{\perp}\right| ; g\right)\right|^{2} \\
& \cdot \delta\left(k+k_{1}+g-2 k_{e}\right) \delta\left(\mathbf{k}_{1}+\mathbf{k}+\mathbf{g}\right) \frac{d \mathbf{k}}{k} \frac{d \mathbf{k}_{1}}{k_{1}} \frac{d \mathbf{g}}{g} .
\end{aligned}
$$


According to the momentum conservation of the reaction products, $\boldsymbol{g}=-\left(\boldsymbol{k}+\boldsymbol{k}_{1}\right)$ and, hence,

$$
\cos \theta_{g}=-\frac{k \cos \theta_{k}+k_{1} \cos \theta_{k_{1}}}{\left|\mathbf{k}+\mathbf{k}_{1}\right|},
$$

where $\theta_{k}$ and $\theta_{k_{1}}$ are polar angles of the photon wave vectors $\boldsymbol{k}$ and $\boldsymbol{k}_{1}$, respectively.

Integrating over the massless boson momentum $\boldsymbol{g}$, the cross-section (71) takes the following form:

$$
\begin{aligned}
d \sigma & =2^{-9} \pi^{-3} r_{e}^{2} \frac{v_{e}}{c} \\
& \cdot \frac{\left(k \cos \theta_{k}+k_{1} \cos \theta_{k_{1}}\right)^{2}}{\left(2 k_{e}-k-k_{1}\right)^{3}} \mid \chi\left(\left|\mathbf{k}_{\perp}-\mathbf{p}_{\perp}\right| ; 2 k_{e}-k\right. \\
& \left.-k_{1}\right)+\left.\chi\left(\left|\mathbf{k}_{\perp}+\mathbf{p}_{\perp}\right| ; 2 k_{e}-k-k_{1}\right)\right|^{2} \delta\left(k+k_{1}\right. \\
& \left.+\left|\mathbf{k}_{1}+\mathbf{k}\right|-2 k_{e}\right) k k_{1} d k d k_{1} d \Omega_{\mathbf{k}} d \Omega_{\mathbf{k}_{1}} .
\end{aligned}
$$

Here $d \Omega_{\boldsymbol{k}}$ and $d \Omega_{\boldsymbol{k}_{1}}$ are the solid angle elements of the photon wave vectors.

Now it is easy to carry out the integration over the energy of one of the two photons, for example, over $k_{1}$. As a result, from (73), we obtain

$$
\begin{gathered}
\frac{\partial^{2} \sigma}{\partial \Omega_{\mathbf{k}} \partial \Omega_{\mathbf{k}_{1}}}=2^{-8} \pi^{-3} r_{e}^{2} \frac{v_{e}}{c} \\
\cdot \int_{0}^{k_{e}} \frac{k_{e} k\left(k_{e}-k\right) d k}{\left(2 k_{e}^{2}-k\left(2 k_{e}-k\right)(1-\cos \vartheta)\right)^{2}} \\
\cdot\left(k \cos \theta_{k}+\frac{2 k_{e}\left(k_{e}-k\right)}{2 k_{e}-k(1-\cos \vartheta)} \cos \theta_{k_{1}}\right)^{2} \\
\cdot\left|\chi\left(\left|\mathbf{k}_{\perp}-\mathbf{p}_{\perp}\right| ; g\right)+\chi\left(\left|\mathbf{k}_{\perp}+\mathbf{p}_{\perp}\right| ; g\right)\right|^{2} .
\end{gathered}
$$

Here we use the following notations: $g$ is the kinetic energy of the massless composite boson emitted,

$$
g=\frac{2 k_{e}^{2}-k\left(2 k_{e}-k\right)(1-\cos \vartheta)}{2 k_{e}-k(1-\cos \vartheta)},
$$

$d \Omega_{\boldsymbol{k}}=\sin \theta_{k} d \theta_{k} d \varphi_{k}, d \Omega_{k_{1}}=\sin \theta_{k_{1}} d \theta_{k_{1}} d \varphi_{k_{1}}, \vartheta$ is the angle between the wave vectors $\boldsymbol{k}$ and $\boldsymbol{k}_{1}\left(\boldsymbol{k} \boldsymbol{k}_{1}=k k_{1} \cos \vartheta\right)$ :

$$
\cos \vartheta=\cos \theta_{k} \cos \theta_{k_{1}}+\sin \theta_{k} \sin \theta_{k_{1}} \cos \left(\varphi_{k}-\varphi_{k_{1}}\right),
$$

and $\varphi_{k}$ and $\varphi_{k_{1}}$ are the azimuthal angles of the wave vectors $\boldsymbol{k}$ and $\boldsymbol{k}_{1}$.

According to (67), the component of the photon wave vector $\boldsymbol{k}$, which is perpendicular to $\boldsymbol{g}$, is given by

$$
\mathbf{k}_{\perp}=\mathbf{k}-\frac{\left(\mathbf{k}\left(\mathbf{k}+\mathbf{k}_{1}\right)\right)}{\left|\mathbf{k}+\mathbf{k}_{1}\right|^{2}} *\left(\mathbf{k}+\mathbf{k}_{1}\right)
$$

and its modulus depends only on $k$ and the angle $\vartheta$,

$$
k_{\perp}=\frac{2 k k_{e}\left(k_{e}-k\right) \sqrt{1-\cos ^{2} \vartheta}}{2 k_{e}^{2}-k\left(2 k_{e}-k\right)(1-\cos \vartheta)} .
$$

3.6. The Observables. Consider colliding two bunches of electrons and positrons with flux densities $I_{-}$and $I_{+}$that are moving towards each other. Let $I_{-}=I_{+}=I$, the spatial lengths of bunches $L$ and their cross-sectional areas $S$ are identical. Then the number of coincidence events per time unit in which one photon is detected in the small element of solid angle $\Delta \Omega_{k}$, and the second photon is in $\Delta \Omega_{k_{1}}$ has the following form:

$$
\frac{\Delta N}{\Delta t}=\zeta(\cos \vartheta) L S I^{2} \Delta \Omega_{\mathbf{k}} \Delta \Omega_{\mathbf{k}_{1}},
$$

where the small solid angles are determined by the angular resolution of an experimental setup, and, according to (74),

$$
\begin{gathered}
\zeta(\cos \vartheta)=2^{-8} \pi^{-3} \frac{r_{e}^{2}}{c} \\
\cdot \int_{0}^{k_{e}} \frac{k_{e} k\left(k_{e}-k\right) d k}{\left(2 k_{e}^{2}-k\left(2 k_{e}-k\right)(1-\cos \vartheta)\right)^{2}} \\
\left(k \cos \theta_{k}+\frac{2 k_{e}\left(k_{e}-k\right)}{2 k_{e}-k(1-\cos \vartheta)} \cos \theta_{k_{1}}\right)^{2} \\
\cdot\left|\chi\left(\left|\mathbf{k}_{\perp}-\mathbf{p}_{\perp}\right| ; g\right)+\chi\left(\left|\mathbf{k}_{\perp}+\mathbf{p}_{\perp}\right| ; g\right)\right|^{2} .
\end{gathered}
$$

That is, precisely the value (80) that has dimension $M \times S$ can be measured in experiments.

Now we carry out the analysis of the observables (74) and (80). If both of these values would only depend on the angle $\vartheta$ between the photon vectors $\boldsymbol{k}$ and $\boldsymbol{k}_{1}$, then we could say that there is the angle symmetry of the twophoton correlation spectra. This symmetry, as far as we know, is experimentally observed for the positron annihilation in solid targets. However, this appears to be due to the fact that the positron energy and momentum relaxations are more rapid processes compared with the annihilation process in solids.

We draw attention to the two effects. The first is that the observables (74) and (80) depend on the momentum of free particles, and, hence, on the initial energy of the electrons and positrons. Then we can argue that the $\boldsymbol{p}_{\perp}$ dependence of the observables will result in the first contribution to the asymmetry of the two-photon angular correlation spectra.

The second effect is due to the radiation pattern of the massless bosons because, according to (71), the cross-section is proportional to $\cos ^{2} \theta_{g}$. That is, these bosons are emitted predominantly along the colliding axis of the two bunches. This results in the second contribution to the asymmetry of these spectra.

3.7. Procedure of Numerical Calculations. Due to computational constraints, we were not able to investigate these two effects presented above. The aim of the subsequent part of this work is to find out the characteristic form of the two-photon angular correlation spectra which are due to the presence of the third particle that is the massless boson. We are trying to answer the question: what typical widths of these spectra can be expected for the reaction (62)? 
With this aim, we replace $\cos ^{2} \theta_{g} \rightarrow 1 / 2$. In the lowenergy limit of the initial particles we can use $k_{\perp} \gg p_{\perp}$. In this case the observables (74) and (80) depend only on the angle $\vartheta$ between the wave vectors of the two photons emitted. As a result, the cross-section (74) is reduced to the following form:

$$
\frac{\partial^{2} \sigma}{\partial \Omega_{\mathbf{k}} \partial \Omega_{\mathbf{k}_{1}}}=(4 \pi)^{-3} r_{e}^{2} \frac{v_{e}}{c} G(\cos \vartheta)
$$

Here we use notations:

$$
\begin{aligned}
& G(\cos \vartheta) \\
& \quad=\int_{0}^{k_{e}} F(k, \cos \vartheta)\left|\chi\left(k_{\perp}(k, \cos \vartheta) ; g(k, \cos \vartheta)\right)\right|^{2} d k,
\end{aligned}
$$

where $g$ and $k_{\perp}$ are given by (75) and (78), respectively, and

$$
F=\frac{k_{e} k\left(k_{e}-k\right)}{\left(2 k_{e}-k(1-\cos \vartheta)\right)^{2}} \text {. }
$$

The observable $\zeta$ given by (80) is rewritten as

$$
\zeta(\cos \vartheta)=(4 \pi)^{-3} \frac{r_{e}^{2}}{c} G(\cos \vartheta)
$$

Further it is convenient to introduce dimensionless variables. For nonrelativistic electrons and positrons $k_{e}=\lambda_{e}^{-1}$. Hence, the dimensionless wave function of the transverse motion of the coupled pair is $\lambda_{e}^{-1} * \chi \rightarrow \chi$; the photon and boson energies become $\lambda_{e} k \rightarrow k$ and $\lambda_{e} g \rightarrow g$. As a result, the functions (75), (78), and (83) are dimensionless:

$$
\begin{aligned}
g & =\frac{2-k(2-k)(1-\cos \vartheta)}{2-k(1-\cos \vartheta)} \\
k_{\perp} & =\frac{2 k(1-k) \sqrt{1-\cos ^{2} \vartheta}}{2-k(2-k)(1-\cos \vartheta)} \\
F & =\frac{k(1-k)}{(2-k(1-\cos \vartheta))^{2}} .
\end{aligned}
$$

The observables (81) and (84) are defined by the same function (82) which is rewritten as

$$
\begin{aligned}
G & (\cos \vartheta) \\
& =\int_{0}^{1} F(k, \cos \vartheta)\left|\chi\left(k_{\perp}(k, \cos \vartheta) ; g(k, \cos \vartheta)\right)\right|^{2} d k .
\end{aligned}
$$

The $G$ function is determined by the momentum-space wave function of the transverse motion of the coupled electron-positron pair, $\chi\left(k_{\perp} ; g\right)$. As was found above, for small momenta of the boson, $g \ll m$; this wave function satisfies the homogeneous Fredholm integral equation of the second kind (33) with the non-Fredholm kernel (36)-(37). We were able to calculate the transverse wave function only in this case. It is a fortunate coincidence that the small energies of the massless bosons, $g \ll m$, are of fundamental importance for the reaction (62), as will be demonstrated below.
Firstly, to find the wave functions of the massless composite boson, we used exactly the calculation procedure presented in Section 2.3 with the same $N=4501$. For the given $k$ and $\vartheta$ the value of this wave function $\chi\left(k_{\perp}(k, \cos \vartheta) ; g(k, \cos \vartheta)\right)$ was calculated by formula:

$$
\chi\left(k_{\perp} ; g\right)=\frac{\alpha}{g} \int_{0}^{f_{0}(g)} Q\left(k_{\perp}, f\right) \chi(f ; g) d f .
$$

Finally, to find the observables (81) and (84) for the given angle $\vartheta$, the integration over $k$ in (88) was carried out. The angular dependence of $\zeta(\cos \vartheta)$ gives us the $2 \gamma$ angular correlation spectra for the reaction (62).

3.8. Results. Equation (85) relates the three variables, the boson energy $g$, the photon energy $k$, and the angle $\vartheta$ between the photon wave vectors $\boldsymbol{k}$ and $\boldsymbol{k}_{1}$. The given angle can be realized for different $g$, since the sums $k+k_{1}+g=2$ and $\boldsymbol{k}+\boldsymbol{k}_{1}+\boldsymbol{g}=0$ are only fixed. However, for each angle there is a minimum value of the boson energy which, according to (85), is

$$
g_{\min }=2 \operatorname{tg}\left(\frac{\phi}{2}\right)\left[\left(1+\operatorname{tg}^{2}\left(\frac{\phi}{2}\right)\right)^{1 / 2}-\operatorname{tg}\left(\frac{\phi}{2}\right)\right]
$$

Here we introduce the angle $\phi=\pi-\vartheta$ that will be used below.

Figure 5 presents the photon energy dependencies of the boson energy converted to $\mathrm{keV}$, for three angles $\phi$. For curve $1 \phi=0.146 \mathrm{mrad}$ and $g_{\min }=74.6 \mathrm{eV}$. The minimum on this curve corresponds to the photon energy $k_{*}$, which is not marked in the figure. Obviously, this photon energy is very close to $m c^{2}$. As shown in Figure 5, this minimal energy is equal to $g_{\min }=220 \mathrm{eV}$ for the angle $\phi=0.431 \mathrm{mrad}$ (curve 2) and $g_{\min }=511 \mathrm{eV}$ for the angle $\phi=1 \mathrm{mrad}$ (curve 3 ).

For the given angle $\phi$ and the boson energy $g>g_{\min }$ there is a pair of the photon energy values, as is clearly demonstrated by the horizontal line on Figure 5. One value of the photon energy from this pair is greater than $k_{*}$, and the other is less than $k_{*}$. These paired values of photon energies will contribute to the integral on the right-hand side of (88). We show below that this integral is evaluated in some neighborhood of $k_{*}$.

There are two more functions, namely, $k_{\perp}$, (86), and $F$, (87), which are also important for calculating the observables (81) and (84). For a given angle $\phi$, the function $k_{\perp}$ goes to zero in the limit $k \rightarrow 0$, reaches a maximum, and then vanishes in the limit $k \rightarrow 1$. The dimensionless photon energy that corresponds to the maximum value of $k_{\perp}$ tends to 1 at $\phi \rightarrow 0$.

The photon energy dependencies of $F(k)$ are very close at small angles. They differ in that for different values of the photon energy $2 /(3-\cos (\phi))$ the function $F(k)$ reaches the maximum values $1 / 8(1-\cos (\phi)$ and then goes sharply to zero in the limit $k \rightarrow 1$.

The behavior of $k_{\perp}$ and $F$ at the singular point $\phi=0$ will be analyzed below.

The angular dependence of the observables is determined by the same integral (88). The $k$ dependencies of the integrand function, $F(k,-\cos \phi)\left|\chi\left(k_{\perp}(k,-\cos \phi) ; g(k,-\cos \phi)\right)\right|^{2}$, are demonstrated in Figures 6-8 for three angles $\phi$. 


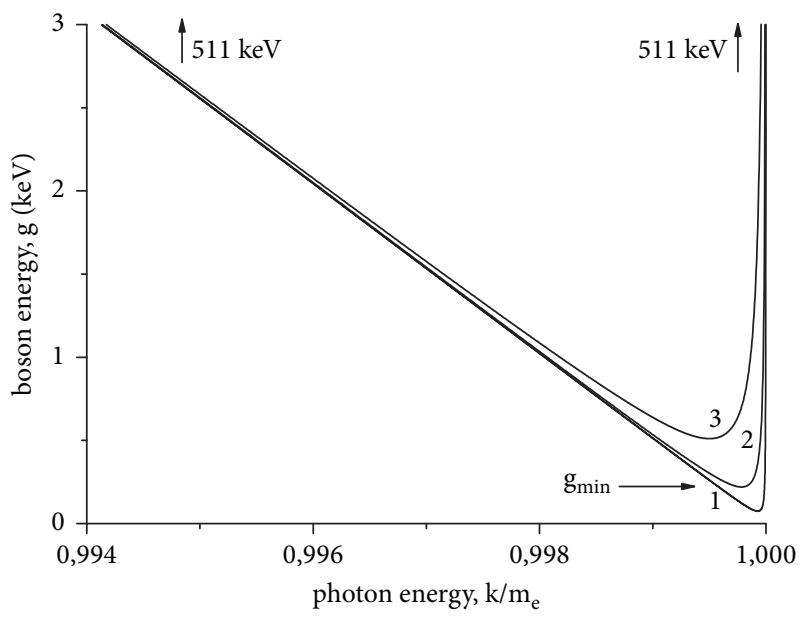

Figure 5: The photon energy dependence of the boson energy at given angle between the photon wave vectors. For the curves from 1 to $3,1: \phi=0.146 \mathrm{mrad} ; 2: \phi=0.431 \mathrm{mrad} ; 3: \phi=1 \mathrm{mrad}$.

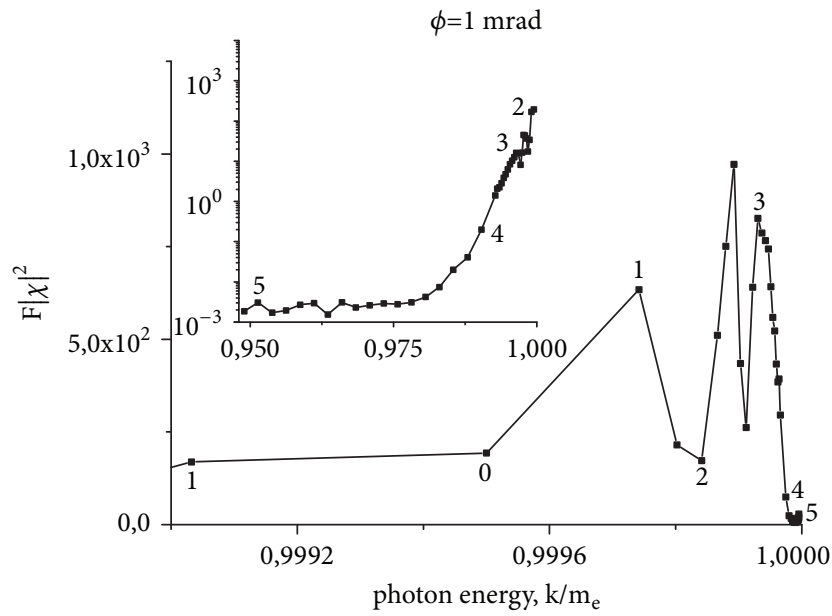

FIgURE 6: The integrand $F|\chi|^{2}$ as a function of the photon energy $k$ for the angle $\phi=1 \mathrm{mrad}$ and $k \geq k_{0}=0.999$. Inset: the same except for $k \leq k_{0}$. For the labeled points the energies of massless bosons are given in the text.

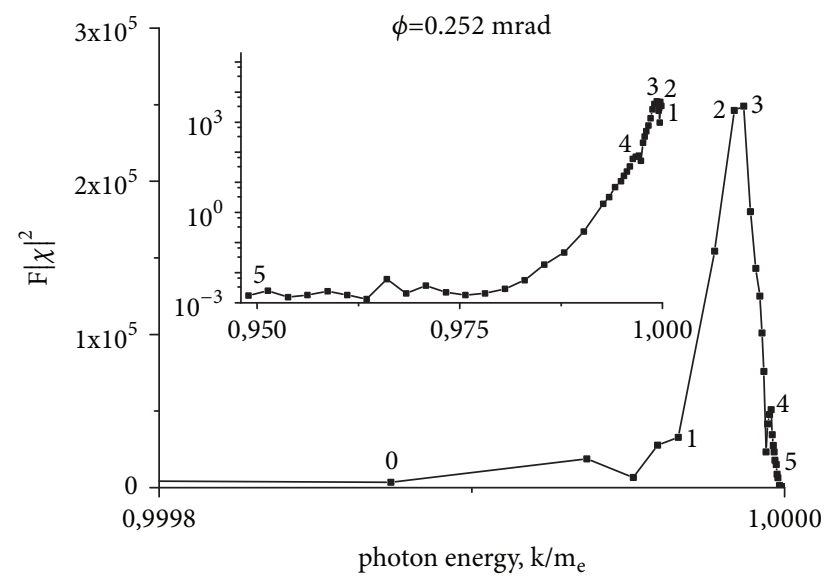

Figure 7: The same as in Figure 6 except for the angle $\phi=0.252$ $\operatorname{mrad}$ and $k_{0}=0.9998$.

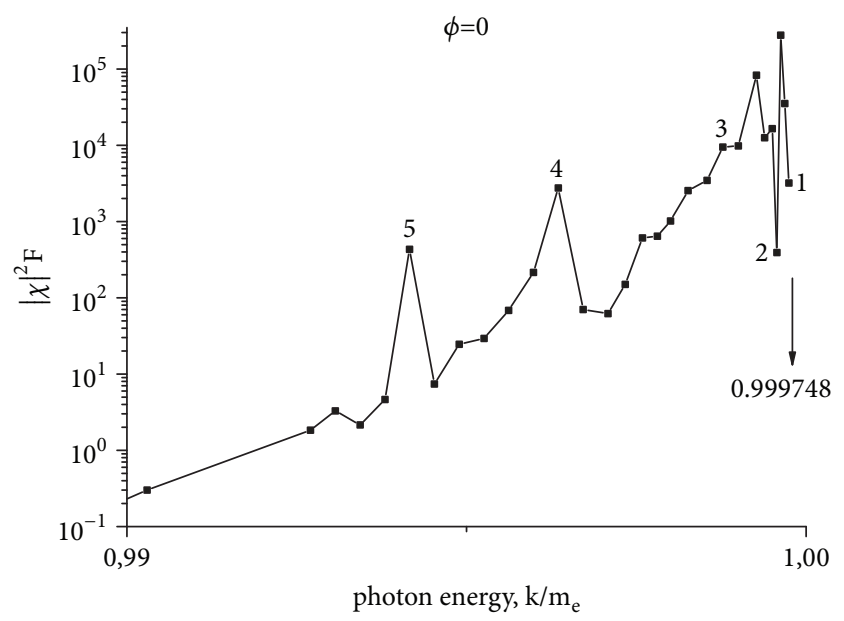

FIGURE 8: The integrand function in (91) for the angle $\phi=0$ in the region of the photon energies $0.99 \leq k \leq 0.999748$.

We begin with the angles $\phi$ which give a very small contribution to the $2 \gamma$ angular correlation spectrum for the reaction (62). Figure 6 presents the integrand as a function of the photon energy $k$ at the angle $\phi=1 \mathrm{mrad}$. As shown above, the transverse wave function $\chi$ is characterized by anharmonic oscillations in the momentum space. As a result, the functions $F|\chi|^{2}$ always represent irregular changes. Except for one point which corresponds to $g_{\min }$, all other points on the curve presented in Figure 6 must be paired, as shown in Figure 5.

The massless boson energies that correspond to the marked points in Figure 6 are as follows: for the point 0 the energy of emitted boson equals $g_{\min }=511 \mathrm{eV}$; for the paired points 1 the boson energy $g=626 \mathrm{eV}$; for $2,884 \mathrm{eV}$; for 3, $1.86 \mathrm{keV}$; for 4, $4.98 \mathrm{keV}$; and for the last pair points 5 , $26.10 \mathrm{keV}$. Thus, the energies of the bosons are much smaller than the electron mass.

For the most right-hand point 5, the dimensionless photon energy differs from unit by $5 \times 10^{-6}$. Therefore, contribution to the integral in the right-hand sides of (88) is very small for the larger photon energies. Using the data presented in Figure 6, we have found that $G=0.40$ at $\phi=1$ mrad.

Figure 7 shows the integrand $F|\chi|^{2}$ as a function of the photon energy $k$ for the angle $\phi=0.252 \mathrm{mrad}$. The characteristic values of this function have significantly increased in comparison with that presented in Figure 6 for the angle $\phi=1 \mathrm{mrad}$.

For the angle $\phi=0.252 \mathrm{mrad}$ the energies of the emitted bosons for the marked points in Figure 7 are as follows: the point 0 corresponds to the energy $g_{\min }=129 \mathrm{eV}$, the paired points 1 correspond to $\mathrm{g}=256 \mathrm{eV} ; 2$ to $511 \mathrm{eV}$; 3 to $626 \mathrm{eV} ; 4$ to $1.86 \mathrm{keV}$; and the last pair points 5 to $26.10 \mathrm{keV}$.

For the most right-hand point 5, the dimensionless photon energy differs from unit by $3 \times 10^{-7}$. Therefore, contribution to the integral in the right-hand sides of (88) is very small for the larger photon energies. We have calculated that $G=11.07$ at $\phi=0.252 \mathrm{mrad}$. 
Note that for the angle $\phi<0.252 \mathrm{mrad}$ the minimal energy of the boson $g_{\min }<129 \mathrm{eV}$ (see Figure 5). Due to computational constraints, we could not find the eigenfunctions of the kernel (36)-(37) for the boson energies significantly less than $130 \mathrm{eV}$. For this reason, in subsequent results calculated for smaller angles $\phi$, the contribution to the $G$ function due to the emission of massless bosons with energies less than $129 \mathrm{eV}$ is not taken into account. Obviously, this will lead to some underestimation of the $G$ function.

As discussed above, the singular point of the integral (88) is the angle $\vartheta=\pi$. It means that $\phi=0$, and, in the reaction (62), along with the boson the two photons having the different energies are emitted at $180^{\circ}$ to each other. According to (85), (86), and (87), in this case, $g=1-k$, $k_{\perp}=0$, and $F=k / 4(1-k)$. Then the integral (88) takes the following form:

$$
G(-1)=\int_{0}^{1} \frac{k d k}{4(1-k)}|\chi(0 ; 1-k)|^{2},
$$

where the function $\chi(0 ; 1-k)$ is found from the equation

$$
\begin{aligned}
& \chi(0 ; 1-k)=\frac{2 \alpha}{1-k} \int_{0}^{\infty}\left(\frac{\Theta(f-1)}{\sqrt{f^{2}-1}}+i \frac{\Theta(1-f)}{\sqrt{1-f^{2}}}\right) \\
& \cdot f \chi(f ; 1-k) d f
\end{aligned}
$$

where $\Theta$ is the Heaviside step function.

Note that in the limit $k \rightarrow 1$ the logarithmic divergence in (91) is absent, since the function $\chi(f, 0)$ vanishes for any $f$. It is due to the fact that the massless boson can not be in the state of rest $(g \neq 0)$.

The integrand in (91) is presented in Figure 8 for the photon energy $k \geq 0.99$. We do not show this function in the region $0.948 \leq k \leq 0.99$ where it gives very small contribution to $G(-1)$. The point labeled 1 corresponds to the energy of the massless boson $g=129 \mathrm{eV}$. In the remaining marked points the boson energies are as follows: for point 2 , $220 \mathrm{eV}$; for 3, $626 \mathrm{eV}$; for 4, $1.86 \mathrm{keV}$; and for 5, $2.98 \mathrm{keV}$.

The vertical arrow in Figure 8 shows the limitation of the region in which the integration in (91) is carried out, so that the region $0.999748<k<1$ remains unaccounted for. The presented curve in Figure 8 leads to the value $G(-1)=45.72$. Of course, the value is underrated because events in which the products of the reaction (62) are the massless bosons with energies less than $129 \mathrm{eV}$ were not taken into account.

Summarizing all the results obtained in this study, we have found the $2 \gamma$ angular correlation spectrum shown in Figure 9. Kinks on the line at $\phi \simeq \pm 0.22 \mathrm{mrad}$ indicate the boundary of the small-angle region with the underestimated data for this spectrum. The reason for this underestimation was discussed above. It can be seen that for the reaction (62) the $2 \gamma$ angular correlation spectrum is characterized by a narrow peak with the full-width-at-half-maximum (FWHM) not exceeding $0.2 \mathrm{mrad}$.

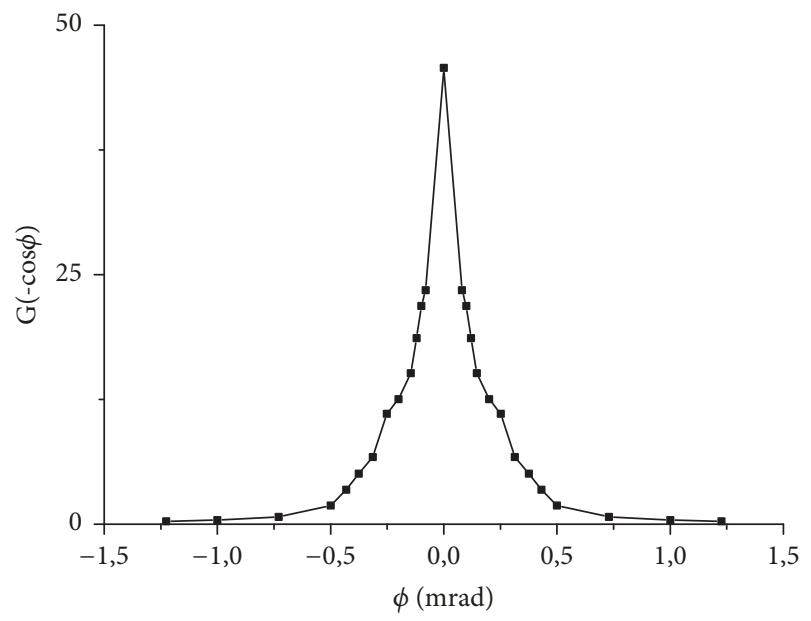

FIGURE 9: The $2 \gamma$ angular correlation spectrum for the reaction (62). The angle $\phi=\pi-\vartheta$.

\section{Conclusion}

In the present work we treated the electron and positron as independent particles, each being characterized by the complete set of the Dirac plane waves. Although this treatment is beyond the standard QED theory, it does not contradict the free particle Dirac theory because, at least, the electron and positron are fermions. This approach leads inevitably to another choice of the free particle propagator as compared to that being currently used in QED. Then the boundstate Bethe-Salpeter equation was investigated in the ladder approximation with these free propagators. The branch of the massless composite bosons formed by the bound electronpositron pairs with the actual coupling equal to the fine structure constant states has been found.

In accordance with the central particle-antiparticle concept in QED, products of the low-energy electron-positron annihilation are just a few photons, and nothing remains of the electron and positron. The singlet pair of the free particles with the center of mass at rest is, with the greatest probability, converted into two photons, which, due to the momentum conservation, should be emitted at the angle of $180^{\circ}$ to each other. The treatment of the electron and positron as independent particles leads to the reaction (62) in which together with emitted photons the reaction products involve the massless boson formed by the coupled electron and positron pair. For this reaction the $2 \gamma$ correlation spectra must always have the finite angular width since the three particles are emitted. That is, the presence of the width is fundamental. As it was found for the new reaction with three product particles, for the extremely nonrelativistic initial particles, the two-photon angular correlation spectrum is characterized by a narrow line with the full-width-at-halfmaximum (FWHM) not exceeding 0.2 mrad.

In many known experiments on the low-energy positron annihilation in condensed and gaseous matters (see [34, $36,38-40$ ] and references therein) the center of mass of annihilating pairs is usually in motion with respect to an observer. Then the angle between the photon directions 
departs from $180^{\circ}$ by an amount of the order of $v_{\mathrm{cm}} / c$, where $v_{\mathrm{cm}}$ is the velocity of the center of mass and $c$ is the velocity of light, and, hence, the annihilation angular correlation spectra of the $2 \gamma$ radiation are characterized by finite widths as well. Obviously, in these experiments it is not possible to distinguish the reaction (62) from the conventional annihilation. In the initial state (left-hand side of (62)) the electron and positron can occur in a parapositronium state that defines other channels of this process.

We argue that it is possible to determine which of the two discussed reactions actually takes place. For this purpose, experiments with colliding beams of extremely nonrelativistic electrons and positrons should be carried out. To prove the reaction (62), parameters of the colliding beams are very important. The ideal would be monoenergetic colliding beams of the annihilating particles with the center of mass at rest. However, the spectra always have finite widths, $\delta \varepsilon$, which can be considered to be the same in both the electron and positron beams. Then, it is easy to estimate that for the conventional annihilation in vacuum the typical width of the $2 \gamma$ angular spectrum is of the order of

$$
\phi_{r} \simeq \frac{v_{e}}{c} \frac{\delta \varepsilon}{\varepsilon}
$$

where $v_{e}$ and $\varepsilon$ are the average velocity and energy of the particles.

For the reaction (62) we found that the angular width of the two-photon correlation spectrum is of the order of $10^{-4} \mathrm{rad}$. Then one can estimate the required parameters of the beams:

$$
\frac{v_{e}}{c} \frac{\delta \varepsilon}{\varepsilon}<10^{-4}
$$

The angular resolution of two-photon detectors should be less then $\phi_{r}$.

For the electron and positron beams with the average energies $\varepsilon=3 \mathrm{eV}$ and the spectral line widths $\delta \varepsilon=50 \mathrm{meV}$ we obtain $\phi_{r}=0.6 \times 10^{-4}$ that satisfies the required condition. The production of the positron beam with close parameters $\varepsilon=1 \mathrm{eV}$ and $\delta \varepsilon=75 \mathrm{meV}$ (for which the value of $\phi_{r}$ is slightly larger, $\phi_{r}=1.5 \times 10^{-4}$ ) was reported in [35].

At high energies, say even for keV beams (not to mention $\mathrm{MeV}$ beams), it is difficult to obtain such narrow lines with $\delta \varepsilon / \varepsilon \simeq 10^{-4}$.

We did not find in the literature similar experiments with such colliding low-energy beams in vacuum. We emphasize that it is very important to use exclusively nonrelativistic particle beams with energies of a few $\mathrm{eV}$, as discussed in the paper. Namely, for such energies of the particles one can obtain the necessary energy homogeneity of the colliding beams.

Unlike the conventional annihilation, for the reaction (62), the two-photon correlation spectrum has a finite angular width in principle. This can be used to establish the actual process. Consider such colliding beams of the low-energy spin-polarized electrons and positrons and assume that the angular resolution of two-photon coincidence-count detector is less than $\phi_{r}$. In case of observation of a narrow peak, the angular width of which is determined by the instrument resolution, one can conclude that only the conventional annihilation takes place, the positron is the antiparticle of the electron, and, respectively, our approach that the electron and positron are independent particles is not correct. If the angular width of the $2 \gamma$ correlation spectrum will be greater than the instrument resolution that should be $<10^{-4} \mathrm{rad}$, then it will mean that the composite massless bosons formed by the coupled electron-positron pairs do really exist, and the reaction (62) does occur.

\section{Conflicts of Interest}

The author declares that he has no conflicts of interest.

\section{References}

[1] R. P. Feynman, "The theory of positrons," Physical Review A: Atomic, Molecular and Optical Physics, vol. 76, no. 6, pp. 749759, 1949.

[2] R. P. Feynman and S. Weinberg, Elementary Particles And the Laws of Physics, Cambridge University Press, 1999.

[3] P. A. M. Dirac, The Principles of Quantum Mechanics, Oxford, Oxford, UK, 4th edition, 1958.

[4] A. I. Akhiezer and V. B. Berestetskii, Quantum Electrodynamics, Wiley, New York, NY, USA, 2nd edition, 1965.

[5] V. B. Berestesky, E. M. Lifshits, and L. P. Pitaevsky, Quantum electrodynamics, Fizmatlit, Moscow, Russia, 2002.

[6] Ya. B. Zeldovich and V. S. Popov, "Electronic structure of superheavy atoms," Soviet Physics Uspekhi, vol. 14, no. 6, p. 673, 1972.

[7] A. B. Migdal, "Vacuum polarization in strong fields and pion condensation," Soviet Physics Uspekhi, vol. 20, p. 879, 1977.

[8] Ya. B. Zeldovich, "Vacuum theory: a possible solution to the singularity problem of cosmology," Soviet Physics Uspekhi, vol. 24 , p. $216,1981$.

[9] I. E. Tamm, "Über die Wechselwirkung der freien Elektronen mit der Strahlung nach der Diracsehen Theorie des Elektrons und nach der Quantenelektrodynamik," Zeitschrift für Physik, vol. 62 , pp. 545-568, 1930.

[10] A. Ore and J. L. Powell, "Three-photon annihilation of an electron-positron pair," Physical Review A: Atomic, Molecular and Optical Physics, vol. 75, no. 11, pp. 1696-1699, 1949.

[11] G. J. Feldman and M. L. Perl, "Electron-positron annihilation above $2 \mathrm{GeV}$ and the new particles," Physics Reports, vol. 19, pp. 233-293, 1975.

[12] J. D. Bjorken and S. J. Brodsky, "Statistical model for electronpositron annihilation into hadrons," Physical Review D: Particles, Fields, Gravitation and Cosmology, vol. 1, no. 5, pp. 1416$1420,1970$.

[13] S. D. Drell, D. J. Levy, and T. Yan, “Theory of deep-inelastic lepton-nucleon scattering and lepton-pair annihilation processes. Iii. deep-inelastic electron-positron annihilation," Physical Review, vol. 1, no. 6, pp. 1617-1639, 1970.

[14] B. Aubert et al., "(BABAR Collaboration), study of $e^{+} e \rightarrow p \bar{p}$ using initial state radiation with BABAR," Physical Review D, vol. 73, Article ID 012005, 2006.

[15] F. J. Gilman and S. H. Rhie, "Calculation of exclusive decay modes of the tau," Physical Review D: Particles, Fields, Gravitation and Cosmology, vol. 31, no. 5, pp. 1066-1073, 1985. 
[16] A. Zee, "Electron-Positron Annihilation in Stagnant Field Theories," Physical Review D: Particles, Fields, Gravitation and Cosmology, vol. 8, no. 11, pp. 4038-4041, 1973.

[17] R. Seidl, I. Adachi, and H. Aihara, "(Belle Collaboration), Invariant-mass and fractional-energy dependence of inclusive production of dihadrons in $e^{+} e$ annihilation at $\sqrt{s}=10.58 \mathrm{Gev}$," Physical Review D, vol. 96, Article ID 032005, 2017.

[18] M. Artuso et al., "(CLEO Collaboration), Charm meson spectra in $e^{+} e$ annihilation at $10.5 \mathrm{GeV}$ center of mass energy," Physical Review D, vol. 70, Article ID 112001, 2004.

[19] H. A. Bethe and E. E. Salpeter, "A relativistic equation for bound-state problems," Physical Review A: Atomic, Molecular and Optical Physics, vol. 84, p. 1232, 1951.

[20] M. Gell-Mann and F. Low, "Bound states in quantum field theory," Physical Review A: Atomic, Molecular and Optical Physics, vol. 84, no. 2, pp. 350-354, 1951.

[21] W. Greiner and J. Reinhardt, "Quantum electrodynamics," Quantum Electrodynamics, pp. 1-447, 2009.

[22] J. S. Goldstein, "Properties of the Salpeter-Bethe Two-Nucleon Equation," Physical Review A: Atomic, Molecular and Optical Physics, vol. 91, no. 6, pp. 1516-1524, 1953.

[23] N. Nakanishi, "A general survey of the theory of the BetheSalpeter equation," Progress of Theoretical and Experimental Physics, vol. 43, 81 pages, 1969.

[24] N. Seto, "Review of the spinor-spinor bethe-salpeter equation. spectral properties in the massless ladder model," Progress of Theoretical Physics Supplements, vol. 95, pp. 25-45, 1988.

[25] A. Nishimura and K. Higashijima, "Exact solutions of the spinor-spinor bethe-salpeter equation and their gauge dependence," Progress of Theoretical Physics, vol. 56, no. 3, p. 908918, 1976.

[26] L. G. Suttorp, "Numerical solution of the spinor Bethe-Salpeter equation and the Goldstein problem," Annals of Physics, vol. 113, no. 2, pp. 257-276, 1978.

[27] W. Lucha, H. Rupprecht, and F. F. Schöberl, "Relativistic treatment of fermion-antifermion bound states," Physical Review D: Particles, Fields, Gravitation and Cosmology, vol. 44, no. 1, pp. 242-249, 1991.

[28] K. Ladanyi, "Spinor bethe-salpeter equation with generalized ladder kernels and the goldstein problem," Annals of Physics, vol. 130, pp. 427-460, 1980.

[29] M. Bawin, J. Cugnon, and H. Sazdjian, "Strong coulomb coupling in the todorov equation," International Journal of Modern Physics A, vol. 11, no. 30, p. 5303, 1996.

[30] H. Sazdjian, "The massless bound state formalism in twoparticle relativistic quantum mechanics," International Journal of Modern Physics A, vol. 3, p. 1235, 1988.

[31] V. Ivanenko, "The preface to the book," in The Latest Development of Quantum Electrodynamics, D. D. Ivanenko, Ed., Foreign Literature Publishing House, Moscow, Moscow, Russia, 1954.

[32] R. P. Feynman, "Space-time approach to quantum electrodynamics," Physical Review A: Atomic, Molecular and Optical Physics, vol. 76, no. 6, pp. 769-789, 1949.

[33] A. P. Prudnikov, Y. A. Brychkov, and O. I. Marichev, Integrals and Series, Special Functions, vol. 2, Fizmatlit, Moscow, Russia, 2003.

[34] M. J. Puska and R. M. Nieminen, "Theory of positrons in solids and on solid surfaces," Reviews of Modern Physics, vol. 66, no. 3, article 841, 1994.

[35] K. G. Lynn, A. P. Mills, R. N. West, S. Berko, K. F. Canter, and L. O. Roellig, "Positron or Positroniumlike Surface State on
Al(100)?” Physical Review Letters, vol. 54, no. 15, pp. 1702-1705, 1985.

[36] P. Coleman, Positron Beams and Their Applications, World Scientific, Singapore, Singapore, 1999.

[37] P. W. Zitzewitz, J. C. Van House, A. Rich, and D. W. Gidley, "Spin Polarization of Low-Energy Positron Beams," Physical Review Letters, vol. 43, no. 18, pp. 1281-1284, 1979.

[38] G. F. Gribakin, J. A. Young, and C. M. Surko, "Positronmolecule interactions: resonant attachment, annihilation, and bound states," Reviews of Modern Physics, vol. 82, no. 3, pp. 2557-2607, 2010.

[39] R. W. Bickmore, "On focusing electromagnetic radiators," Canadian Journal of Physics, vol. 35, no. 11, pp. 1292-1298, 1957.

[40] M. Charlton and J. W. Humberston, Positron Physics, Cambridge University Press, Cambridge, UK, 2000. 

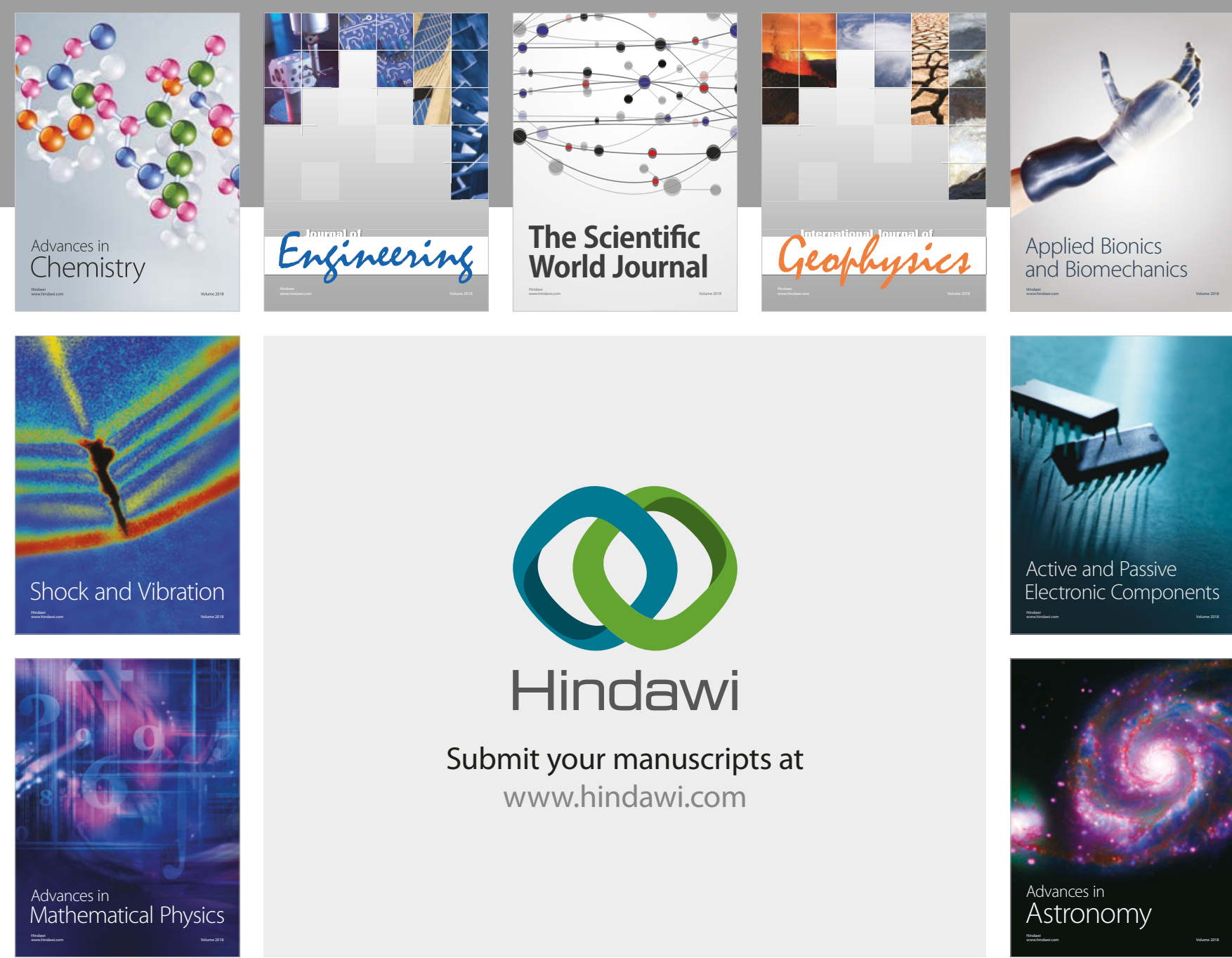

Submit your manuscripts at

www.hindawi.com

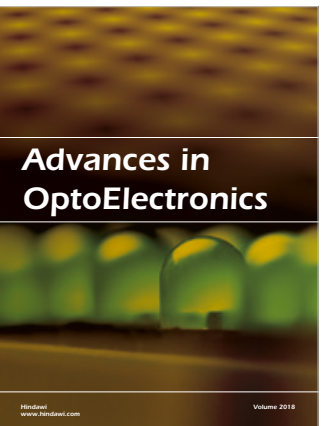

\section{Rotcting Machinery}
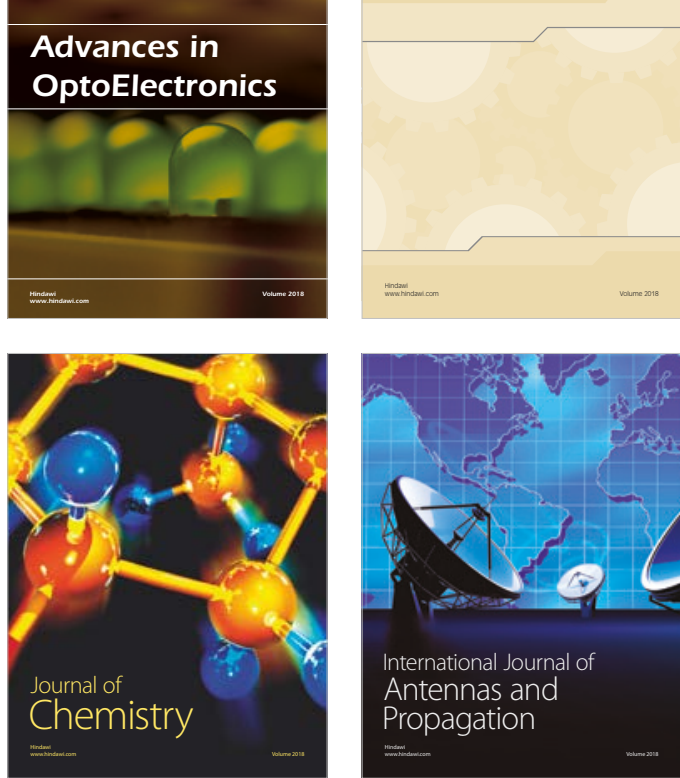

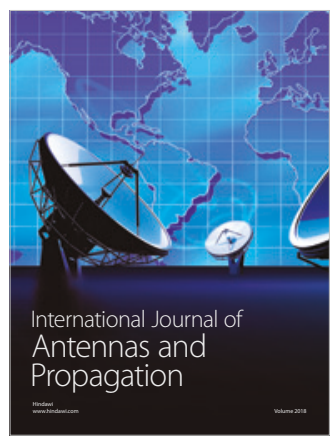

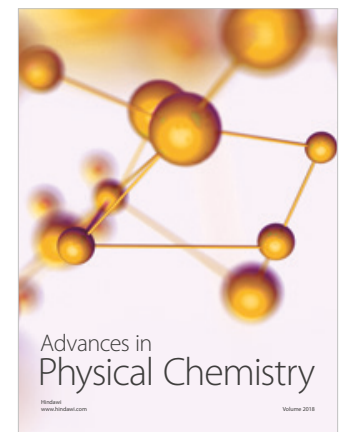

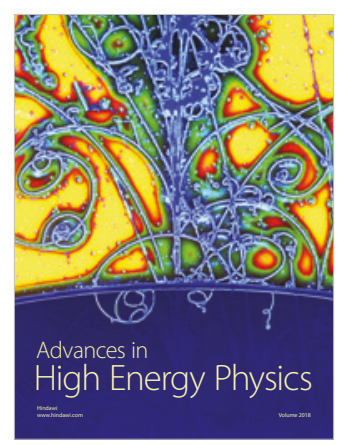

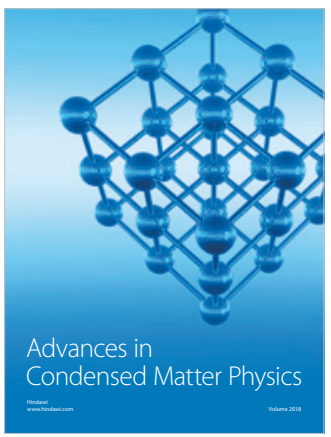

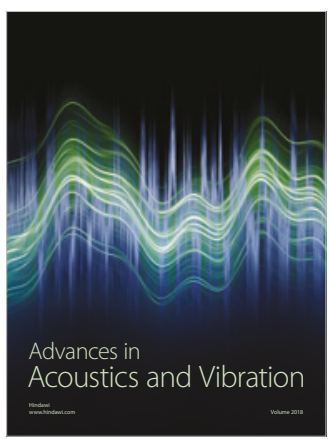

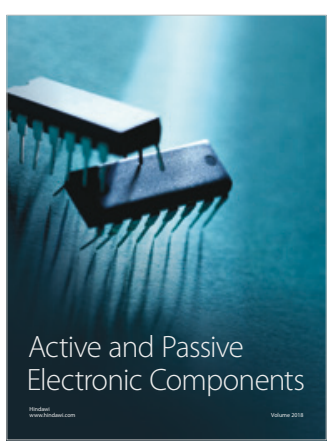
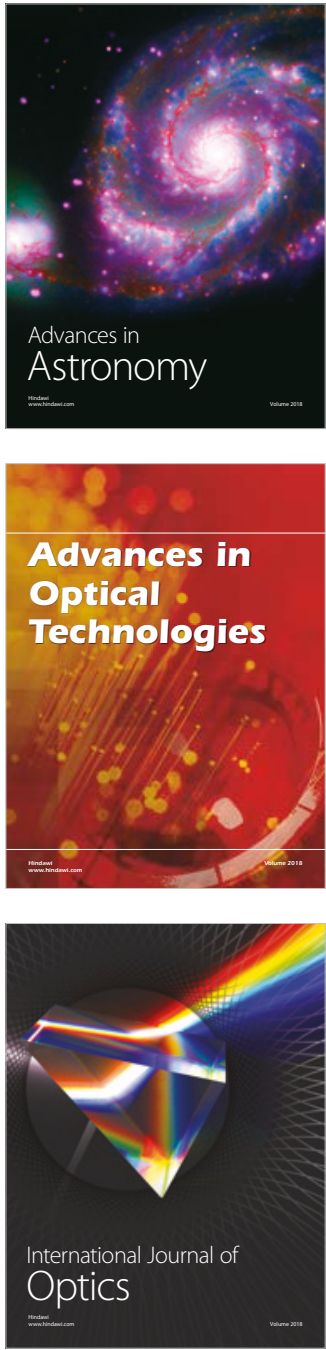